\title{
25. RADIOLARIA FROM THE WEST-CENTRAL INDIAN OCEAN AND GULF OF ADEN, DSDP LEG 24
}

\author{
Annika Sanfilippo and W. R. Riedel, Scripps Institution of Oceanography, La Jolla, California
}

\section{INTRODUCTION}

Preparations of radiolarians and preliminary shipboard interpretations were made during Leg 24 by David C. Clegg. This chapter includes both the results of his preliminary work and our more detailed interpretations made ashore. Shortage of time has prevented our investigating this material thoroughly, but since the late Neogene radiolarians are better represented than in other legs that we have investigated, they are treated in somewhat greater detail. In this connection we have examined the late Neogene radiolarian sequences of the eastern tropical Pacific Sites 73 and 77B (from Legs 8 and 9, respectively) in order to check whether the stratigraphic order of events (earliest and latest occurrences of species) observed in the Indian Ocean material is similar to that observed in the distant tropical Pacific.

In addition to the late Neogene that we describe herein, good radiolarian sequences obtained by this leg of the drilling expedition include a long Miocene sequence at Site 238 and middle Eocene at Site 237.

Of the drilling sites occupied during Leg 24 , the following ones yielded significant numbers of radiolarians:

Site $231-11^{\circ} 53.41^{\prime} \mathrm{N}, 48^{\circ} 14.71^{\prime} \mathrm{E}$; water depth 2161 meters

Site $232-14^{\circ} 28.93^{\prime} \mathrm{N}, 51^{\circ} 54.87^{\prime} \mathrm{E}$; water depth 1758 meters

Site $233-14^{\circ} 19.68^{\prime} \mathrm{N}, 52^{\circ} 08.11^{\prime} \mathrm{E}$; water depth 1860 meters

Site $234-04^{\circ} 28.96^{\prime} \mathrm{N}, 51^{\circ} 13.48^{\prime} \mathrm{E}$; water depth 4738 meters

Site $235-03^{\circ} 14.06^{\prime} \mathrm{N}, 52^{\circ} 41.64^{\prime} \mathrm{E}$; water depth 5146 meters

Site $236-01^{\circ} 40.62^{\prime} \mathrm{S}, 57^{\circ} 38.85^{\prime} \mathrm{E}$; water depth 4504 meters

Site $237-07^{\circ} 04.99^{\prime} \mathrm{S}, 58^{\circ} 07.48^{\prime} \mathrm{E}$; water depth 1640 meters

Site $238-11^{\circ} 09.21^{\prime} \mathrm{S}, 70^{\circ} 31.56^{\prime} \mathrm{E}$; water depth 2844 meters

The Pacific sites from which late Neogene radiolarians were investigated are:

Site $73-01^{\circ} 54.58^{\prime} \mathrm{S}, 137^{\circ} 28.12^{\prime} \mathrm{W}$; water depth 4387 meters

Site $77-00^{\circ} 28.90^{\prime} \mathrm{N}, 133^{\circ} 13.70^{\prime} \mathrm{W}$; water depth 4291 meters

For the sites at which radiolarians are adequate for stratigraphic interpretation, species present in each sample are tabulated (Tables 1-9). For sites with radiolarians insufficient for stratigraphic interpretation, a paragraph describes the occurrences in general terms. A list of radiolarian events (earliest and latest occurrences of taxa) arranged stratigraphically (Table 12) and a summary range chart (Figure 2) are presented for the late Neogene only.
The form of presentation of these results follows closely that used in the radiolarian report for DSDP Leg 15 (Riedel and Sanfilippo, in press). There is no systematic section, its place being taken by a species list (which includes, however, descriptions of a new subspecies and three new species, one of them in the new genus Bekomiforma).

\section{OCCURRENCES OF RADIOLARIANS}

The following paragraphs briefly describe the radiolarian occurrences, and Figure 1 summarizes this information in relation to the occurrences of calcareous microfossils. In the tabulations of species occurrences (Tables 1-11) and in the paragraphs below, the following abbreviations and signs are used: A (abundant), C (common), R (rare), + (very rare), E (excellently preserved), G (well preserved), M moderately preserved), P (poorly preserved), ? (doubtful identification), ! (believed to be reworked from older sediments, or caved from younger ),-(looked for, and not found), and X (not found, but absence may be due to some secondary factor such as solution, excessive sieving of the assemblage, etc.).

\section{Site 231}

The only sample with abundant well-preserved radiolarians is $231-1-1,145-147 \mathrm{~cm}$, which is Quaternary in age. Below that, most of the samples from Core 2 to Core 16 contain radiolarians, which are, however, somewhat dissolved and therefore inadequate for biostratigraphic interpretation.

Radiolarian abundances and preservation in all samples examined from this site are as follows: $231-1-1,145-147 \mathrm{~cm}$ (A, G); -2-2, 49-51 cm (R, M); -3-1, 58-60 cm (R, M);-3-4, $53-55 \mathrm{~cm}(\mathrm{R}, \mathrm{M}) ;-4-1,50-52 \mathrm{~cm}(\mathrm{R}, \mathrm{M}) ;-4-3,50-52 \mathrm{~cm}(\mathrm{R}$, M); $-5-1,49-51 \mathrm{~cm}$ (C, M); -5-6, 50-52 cm (F, M); -5-6, $115-117 \mathrm{~cm}$ (None); -6-1, 50-52 cm (R, M); -6-6, 50-52 cm (F, M); -7-3, 130-132 cm (None); -8-1, 59-61 cm (F, M); $-8-6,110-112 \mathrm{~cm}$ (C, M); -9-1, 49-51 cm (C, M); -9-6, 120-122 cm (None); -10-3, 29-31 cm (C, M); -10-5, 130-132 $\mathrm{cm}(\mathrm{C}, \mathrm{M}) ;-11-2,50-52 \mathrm{~cm}$ (C, M); -11-3, 110-112 cm (C, M); $-12-1,50-52 \mathrm{~cm}(\mathrm{R}, \mathrm{M}) ;-12-4,130-132 \mathrm{~cm}(\mathrm{~F}, \mathrm{M})$; $-13-2,30-32 \mathrm{~cm}$ (F, M); -13-6, 108-110 cm (F, M); -14-1, 49-51 cm (F, M); -14-5, 118-120 cm (F, M); -15-2, 83-85 $\mathrm{cm}(\mathrm{R}, \mathrm{M}) ;-16-2,19-21 \mathrm{~cm}(\mathrm{R}, \mathrm{M}) ;-16-6,119-121 \mathrm{~cm}$ (None); -17-1, 58-60 cm (None); -17-5, 119-121 cm (None); $-18-2,29-31 \mathrm{~cm}$ (None); -19-2, 29-31 cm (F, M); -19-4, $110-112 \mathrm{~cm}$ (None); -20-1, $44-46 \mathrm{~cm}$ (None); -20-6, 119-121 cm (None); -21-1, 59-61 cm (None); -21-6, $109-111 \mathrm{~cm}$ (None); -22-1, 84-86 cm (+, M); -23-6, 119-121 cm (None); -24-5, 119-120 cm (None); -25-6, 120-122 cm (None); -26-6, 110-112 cm (None); -27-6, 110-112 cm (R, pyritized); -28-6, 110-112 cm (R, pyritized); -29-6, 110-112 $\mathrm{cm}$ (+, pyritized); $-30-6,110-112 \mathrm{~cm}$ (None); $-31-5$, 
110-112 cm (None); -32-6, 110-112 cm (None); -33-5, $110-112 \mathrm{~cm}$ (None); -34-6, 110-112 cm (None); -35-2, $50-52 \mathrm{~cm}$ (None); -36-6, 100-102 cm (None); -37-5, 100-102 cm (None); -38-4, 60-62 cm (None); -39-4, 100-102 cm (None); $-40-5,104-106 \mathrm{~cm}$ (None); $-41-5$, $120-122 \mathrm{~cm}$ (None); $-42-6,120-122 \mathrm{~cm}$ (None); -43-6, $120-122 \mathrm{~cm}$ (None); -44-2, 110-112 cm (None); -45-6, 40-42 cm (None); -47-1, 60-62 cm (None); -47-6, 120-122 cm (None); -48-1, 119-121 cm (None); -49-2, 40-42 cm (None); -50-6, 110-112 cm (None); -51-6, 110-112 cm (None); -52-5, 130-132 cm (R, M); -53-6, 120-122 cm (R, M); -54-2, 56-58 cm (None); -55-1, 70-72 cm (R, P); -56-2, 30-32 cm (None); -57-1, 107-109 cm (None); -58-6, 50-52 cm (None); -59-6, 102-104 cm (None); -60-2, 70-72 cm (R, pyritized); -61-6, 117-119 $\mathrm{cm}$ (None); and -62-1, 85-90 cm (None).

\section{Site 232 (Table 1)}

Radiolarians are generally few to abundant, and moderately to well preserved, in all samples examined from Hole 232 and the top 10 cores of Hole 232A. Below that they are absent, with the exception of few to abundant, moderately to well preserved specimens in Cores 19 to 21 of Hole 232A.

The base of the Quaternary is uncertain, apparently lying between 232-4-6 and 232-8-3. The entire Pterocanium prismatium Zone is apparently included within this uncertain interval. All samples from 232-8-3 through 232A-6-1 are within the Spongaster pentas Zone, and samples from 232A-7-1 through 232A-21-2 are in the Stichocorys peregrina Zone.

\section{Site 233 (Table 2)}

In all samples examined from Holes 233 and 233A, radiolarians are common to abundant, moderately to excellently preserved.

The base of the Quaternary is between 233-5-6 and 233-7-5, the base of the Pterocanium prismatium Zone is between 233-16-6 and 233-18-5, and the uncertain base of the Spongaster pentas Zone appears to lie below 233A-4-4.

\section{Site 234}

Radiolarians are poorly to moderately preserved, most pyritized, in all samples examined from 234-1-1 through 234-4-6. They are absent from samples examined from 234-5-3 through 234-15-3, with the exception that a sample from 234-7-1 contains few, poorly preserved radiolarians, and 234-15-3 contains very rare, poorly preserved and pyritized radiolarians. The assemblages are not adequate for stratigraphic interpretation. No samples were examined from Hole 234A.

\section{Site 235 (Table 3)}

Samples from 235-1-1 through 235-5-6 contain common to abundant, well to excellently preserved radiolarians, and species occurrences are shown in Table 3. Below this, radiolarians are much less common, generally pyritized, and not sufficiently well preserved for stratigraphic interpretation. Occurrences are as follows: $235-7-1,106-110 \mathrm{~cm}(\mathrm{R}$, M); -7-4, 96-100 cm (R, P); -8-1, 96-100 cm (R, P); -9-1, $57-62 \mathrm{~cm}(\mathrm{R}, \mathrm{P})$; -9-6, 93-99 cm (R, P); -10-1, 50-52 cm (F,
P); -10-6, 101-105 cm (R, P); -11-6, 110-112 cm (F, M); $-12-1$, 93-96 cm (R, P); -12-3, 89-93 cm (C, M); -13-1, $49-51 \mathrm{~cm}(\mathrm{R}, \mathrm{P}) ;-13-3,109-111 \mathrm{~cm}(\mathrm{R}, \mathrm{P}) ;-14-2,34-40 \mathrm{~cm}$ $(\mathrm{R}, \mathrm{P}),-15-1,60-62 \mathrm{~cm}$ (None); -15-4, 108-110 cm (None); $-16-1,136-138 \mathrm{~cm}$ (None).

The section from the top of the hole through the sample at 235-5-1, 68-74 $\mathrm{cm}$ contains Quaternary radiolarians, and the assemblage in $235-5-6,99-103 \mathrm{~cm}$ is in the Pterocanium prismatium Zone.

\section{Site 236 (Tables 4, 5)}

Samples from 236-1-1 through 236-3-1 contain abundant well-preserved radiolarians; those from 236-3-3 through 236-4-5 contain radiolarians progressively fewer and more corroded; those from 236-5-1 through 236-7-6 contain no radiolarians; those from 236-8-1 through 236-14-2 contain rare to common, poorly to moderately well preserved radiolarians; and those from 236-20-2 through 236-33-2 contain rare to abundant, poorly to well-preserved radiolarians. In addition to the samples included in Tables 4 and 5, the following were examined and found to contain no radiolarians: 236-14-3, 109-112 $\mathrm{cm}$; -15-1, 49-52 cm; -15-6, 28-32 cm; -15-6, 94-97 cm; $-16-1,58-60 \mathrm{~cm} ;-16-6,102-105 \mathrm{~cm} ;-17-1,59-62 \mathrm{~cm} ;-17-6$, 98-102 cm; -18-1, 45-50 cm; -18-6, 108-111 cm;-19-1, $88-93 \mathrm{~cm} ;-19-3,95-105 \mathrm{~cm}$; and $-33-3,68-73 \mathrm{~cm}$.

All of the radiolarian assemblages at this site include reworked older radiolarians-often in such high proportions as to obscure the autochthonous component. Therefore the zonal assignments indicated are only tentative and approximate. The upper limit of the Pterocanium prismatium Zone appears to lie approximately between 236-2-1 and 236-3-1. Assemblages from 236-10-2 through 236-14-2 seem probably assignable to the Ommatartus antepenultimus Zone, and those from 236-20-2 through 236-33-2 are approximately in the Theocyrtis tuberosa Zone. A few of the reworked species are tabulated (followed by exclamation marks) to indicate the range of ages involved in the reworking.

\section{Site 237 (Tables 6, 7, and 8)}

Radiolarians in samples from 237-1-1 through 237-12-1 are rare to abundant, moderately to well preserved (Table 6). From 237-25-1 through 237-32-2 they are abundant, and moderately to well preserved (Table 7). From 237-36-1 through $237-42-1$ they are few to common, moderately to well preserved (Table 8). In the interval from 237-13-1 through 237-24-1 and from 237-43-2 through 237-54-1 radiolarians are insufficient for stratigraphic interpretation and therefore are not tabulated. Their occurrences are as follows: $237-13-1,49-52 \mathrm{~cm}(\mathrm{~F}, \mathrm{M}) ;-14-1,49-51 \mathrm{~cm}(\mathrm{C}$, M); -15-1, 129-132 cm (None); -16-1, 50-52 cm (None), $-17-1,49-51 \mathrm{~cm}$ (None); -18-1, 48-51 cm (None); -18-1, 133-135 cm (None); -19-1, 48-51 cm (R, P); -20-1, 48-50 $\mathrm{cm}(\mathrm{R}, \mathrm{P}) ;-21-1,50-52 \mathrm{~cm}(\mathrm{R}, \mathrm{M}) ;-22-1,100-102 \mathrm{~cm}(\mathrm{R}$, P); -23-1, 48-51 cm (R, P); -24-1, 39-43 cm (R, P); -43-2, approx. $105 \mathrm{~cm}(\mathrm{R}, \mathrm{M}) ;-44-1$, approx. $85 \mathrm{~cm}(\mathrm{~F}, \mathrm{M}):-45-1$, approx. $68 \mathrm{~cm}(\mathrm{R}, \mathrm{M}) ;-46-2$, approx. $75-77 \mathrm{~cm}(\mathrm{~F}, \mathrm{P})$; $-47-2$, approx. $66 \mathrm{~cm} \mathrm{(F,} \mathrm{P);} \mathrm{-48-1,} \mathrm{approx.} 98 \mathrm{~cm} \mathrm{(F,} \mathrm{P);}$ -49-2, approx. $90 \mathrm{~cm}(\mathrm{~F}, \mathrm{P}) ;-50-1$, approx. $70 \mathrm{~cm}(\mathrm{~F}, \mathrm{P})$; 
-52-1, approx. $105 \mathrm{~cm}(\mathrm{R}, \mathrm{M}) ;-53-2$, approx. $82 \mathrm{~cm}(\mathrm{R}, \mathrm{P})$; -54-1, approx. $120 \mathrm{~cm}(\mathrm{C}, \mathrm{P})$.

Assemblages in 237-1-1 and 237-2-1 are Quaternary, the Pterocanium prismatium Zone may be present though unrecognizable in 237-4-1 and 237-5-1, the Spongaster pentas and Stichocorys peregrina Zones apparently occur in 237-6-1 through 237-11-1, and 237-12-1 is in the Ommatartus penultimus Zone.

Assemblages in 237-25-1 and 237-26-1 are in the Podocyrtis mitra Zone, those in 237-27-1 and probably 237-28-1 are in the Podocyrtis ampla Zone, those in 237-29-1 (probably) and 237-30-1 are in the Thyrsocyrtis triacantha Zone, and that in $237-32-2$ is in the Theocampe mongolfieri Zone.

Assemblages in 237-36-1 and 237-37-1 are probably in the Buryella clinata Zone (the transition from Phormocyrtis striata exquisita to $P$. striata striata, used to define the base of the $P$. striata striata Zone, seems overshadowed by other evidence indicating the Buryella clinata Zone), and those in 237-38-1 through 237-42-1 are in the Bekoma bidarfensis Zone.

\section{Site 238 (Table 9)}

Radiolarians are few to abundant, moderately to well preserved in samples from 238-1-1 through 238-24-1 and not consistently so common and well preserved in samples from 238-24-6 through 238-39-2. In samples from 238-39-6 through 238-54-1, radiolarians are absent except for rare, moderately well preserved specimens in 238-53-2.

The upper limit of the Pterocanium prismatium Zone appears to lie between 238-3-6 and 238-4-1, though the lower evolutionary limit of Theocorythium trachelium appears to be considerably below that level. The lower limit of the Pterocanium prismatium Zone is between 238-8-2 and $238-9-1$, the lower limit of the Spongaster pentas Zone between 238-13-1 and 238-13-5, the lower limit of the Stichocorys peregrina Zone between 238-20-6 and 238-21-1, the lower limit of the Ommatartus penultimus Zone cannot be determined, the lower limit of the Ommatartus antepenultimus Zone is between 238-29-1 and 238-30-2, the lower limit of the Cannartus petterssoni Zone cannot be determined, the lower limit of the Dorcadospyris alata Zone is approximately in 238-38-6 (uncertain because of poor preservation), and the lower limit of the Calocycletta costata Zone is below 238-39-2.

\section{LATE NEOGENE RADIOLARIAN STRATIGRAPHY}

Several of the drilling sites on this leg yielded late Neogene radiolarian sequences more continuous and better preserved than those that we have investigated from earlier legs of DSDP. We have, therefore, investigated them in some detail and compared their succession of radiolarian events with the succession in the eastern tropical Pacific (from Legs 8 and 9, for which Initial Reports have now been published and from which material is now available for detailed investigations). Whereas our earlier efforts have largely been directed toward determining a succession of radiolarian events occurring synchronously throughout the tropical oceans (and to this end we have in earlier papers tended to determine a most probable "average" level of the earliest or latest occurrence of species, evolutionary transitions, etc.), Cenozoic radiolarian stratigraphy has now reached the stage at which it seems profitable to investigate in detail the differences in the sequences of radiolarian events in different biogeographic regions. By doing so, it should eventually be possible to perform correlations more precisely within (and perhaps between) biogeographic regions, and the data gathered should provide insight into the regions in which various species evolved, rates of migration, etc. In order for this to be possible, the majority of radiolarian events must occur in the same sequential order in the different biogeographic regions (so that reliable correlations may be made), and only a minority of the events may be time-transgressive. That this is true in a general way has been amply demonstrated by the utility of the radiolarians for reliable correlations-and confirmation is provided by the fact that in Table 12 most of the correlation lines between the Indian Ocean and Pacific sequences do not cross.

For comparison with the Indian Ocean late Neogene sequence, we have chosen to examine two holes (73 and 77B) from the eastern half of the tropical Pacific, where the late Neogene is characterized by high rates of sediment accumulation and good preservation of siliceous microfossils. Radiolarians in the very late Miocene through Quaternary at these sites are indicated in Tables 10 and 11. The radiolarians in these cores have previously been analyzed in a preliminary fashion by Moore (1971) and Goll (1972), but it is only by reexamining the assemblages that we have been able to ensure that the same concept of each species has been uniformly applied to the several sequences being compared here and that apparent differences in ranges are not due to differences in the species definitions. Comparison of our tabulations with those of the cited authors reveals some differences in the ranges of species and consequently in the location of zonal boundaries.

The events list (Table 12) is arranged to facilitate comparison between the late Neogene sequences of radiolarian events in the western Indian Ocean and the eastern Pacific. The substantial number of parallel correlation lines (including, fortunately, those representing zonal markers) connecting the Indian Ocean and Pacific parts of Table 12 can, with considerable confidence, be interpreted as indicating synchronous levels. Crossing of correlation lines is interpreted to indicate that events occur at different times in the two regions. A simple case is represented by the evolutionary transition from Ommatartus avitus to $O$. tetrathalamus, which is manifested later in the Indian Ocean sequences than in the Pacific sequences investigated. More complicated relations are indicated near the top and near the bottom of Table 12, where correlation lines cross to such an extent that it is difficult to determine which indicate synchronous levels. These data are obviously insufficient to form the basis for significant deductions, but it seems clear that additional detailed work along these lines will be necessary in order to increase the precision and reliability with which radiolarians can be used for correlation.

In the course of obtaining the results discussed above, we have attempted to investigate the stratigraphic distribution of some radiolarians that other authors (e.g., 
Hays, 1970, 1971; Nigrini, 1971, in press; Petrushevskaya, 1972; Goll, 1972; Kling, 1973) have reported as useful in subdividing late Neogene sequences. Below are listed species and their reported changes which we have attempted, without success, to apply stratigraphically. Our lack of success in this endeavour may be due to the fact that we have not pursued subtle changes with sufficient persistence.

Druppatractus aquilonius Hays (1970), reported to evolve from Stylacontarium sp. aff. S. bispiculum Popofsky (Kling, 1973) by the outer medullary shell becoming ellipsoidal.

Lamprocyclas heteroporos Hays (1965) has subtle morphotypic limits difficult to apply.

Stylatractus universus Hays (1970)-Axoprunum angelinum (Campbell and Clark) of Kling (1973)-has an upper limit difficult to determine.

Buccinosphaera invaginata Haeckel, applied stratigraphically by Nigrini (1971), could not be found in our tropical Pacific sequences.

Anthocyrtidium angulare Nigrini (1971) has stratigraphic limits that proved difficult to determine.

Amphirhopalum ypsilon Haeckel is reported by Nigrini (1971) to undergo evoluntionary change and to have evolved from $A$. virchowii (Haeckel) (Nigrini, in press), but we have found the different morphotypes to occur in approximately similar numbers through long periods of time.

Sphaeropyle robusta Kling (1973) is reported to have evolved from $S$. langii Dreyer by increase in size and development of a more robust cortical shell.

The younger of the Tholospyris taxa defined by Goll (1972) are reported to be useful for stratigraphic subdivision of the late Neogene, but are too sparse to be reliable in our sequences.

We have been able to confirm the stratigraphic usefulness of several radiolarian events described by earlier authors. The lower limit of Pterocorys hertwigii morphotypes (Petrushevskaya, 1972) and the evolutionary transition from Theocorythium vetulum to $T$. trachelium (Nigrini, 1971) were found to be approximately synchronous with the extinction of Pterocanium prismatium, and the evolutionary transition from Lamprocyrtis neoheteroporos to L. haysi (Kling, 1973) was found to occur slightly above this level. The earliest occurrence of morphotypes of Nephrospyris renilla was confirmed to be within the Spongaster pentas Zone as reported by Goll (1972), and the latest occurrence of Lychnodictyum audax occurs in the uppermost part of that zone. These events are welcome additions to the sparse list available for long-range correlations of radiolarian sequences of this age.

During the later stages of this investigation, three additional radiolarians were found to show promise for future correlations in the late Neogene. These are described as the new species Spongodiscus ambus, Artostrobium rhinoceros, and Bekomiforma mynx. It has not been practical, at this point in time, to include the limits of their occurrence in the list of events (Table 12); they are, however, indicated approximately on the range chart (Figure 2).

\section{SPECIES LIST}

The purpose of this list is to provide bibliographic references to the taxa mentioned in this chapter. If the published literature contains several different concepts of the limits of a species, the reference cited conforms to the concept as applied here. The list also serves as an index, since it directs the reader to the references to each taxon within this chapter. References are to sections of the text (Sect.), rather than to pages.

Acrobotrys tritubus Riedel, 1957, p. 80, pl. 1, fig. 5 ..

This chapter: Tab. 1-3, 6, 9-12; Text-fig. 2.

Amphicraspedum murrayannum Haeckel-Sanfilippo and Riedel, 1973 , p. 524 , p. 10, figs. 3-6; pl. 28, fig. 1 .

This chapter: Tab. 5,8 .

Amphirhopalum ypsilon Haeckel-Nigrini, 1967, p. 35, p. 3 , figs. 3a-d.

This chapter: Sect. 3.

Artophormis dominasinensis (Ehrenberg)-Riedel and Sanfilippo, 1971, p. 1592, pl. 6, fig. 6 .

This chapter: Tab. 7.

Artophormis gracilis Riedel, 1959, p. 300, pl. 2, figs. 12, 13.

This chapter: Tab. 5.

Artophormis sp. cf. A. gracilis Riedel-Sanfilippo et al., in press. Under this name we do not include the form designated as Artophormis cf. A. gracilis by Moore (1971).

This chapter: Tab. 1, 2; Pl. 2, fig. 2.

Artostrobium auritum (Ehrenberg) group-Riedel and Sanfilippo, 1971, p. 1599, pl. 1H, figs. 5-8.

This chapter: Tab. 1, 2, 9, 11 .

Artostrobium doliolum Riedel and Sanfilippo, 1971, p. 1599 , pl. $1 \mathrm{H}$, figs. 1-3; pl. 8, figs. 14, 15 .

This chapter: Tab. 1-4, 6, 9-12; Text-fig. 2 .

Artostrobium miralestense (Campbell and Clark)-Riedel and Sanfilippo, 1971, p. 1599, pl. 1H, figs. 9-17; pl. 2I, figs. 9, 10; pl. 3E, fig. 12 .

This chapter: Tab. 1-3, 6, 9-11.

Artostrobium rhinoceros Sanfilippo and Riedel, new species Plate 4, Figures 8, 9.

Many-segmented, cylindro-conical form with generally five to seven segments. Cephalis and thorax difficult to distinguish exteriorly, with thick walls, rather irregularly arranged subcircular pores, and tending to have short, broad thorns on the surface. Cephalis bears a strong, short, bladed apical horn and a prominent vertical tube directed obliquely upward, often tending to flare distally and to have a toothed termination. The dorsal bar penetrates the middle or lower part of the 


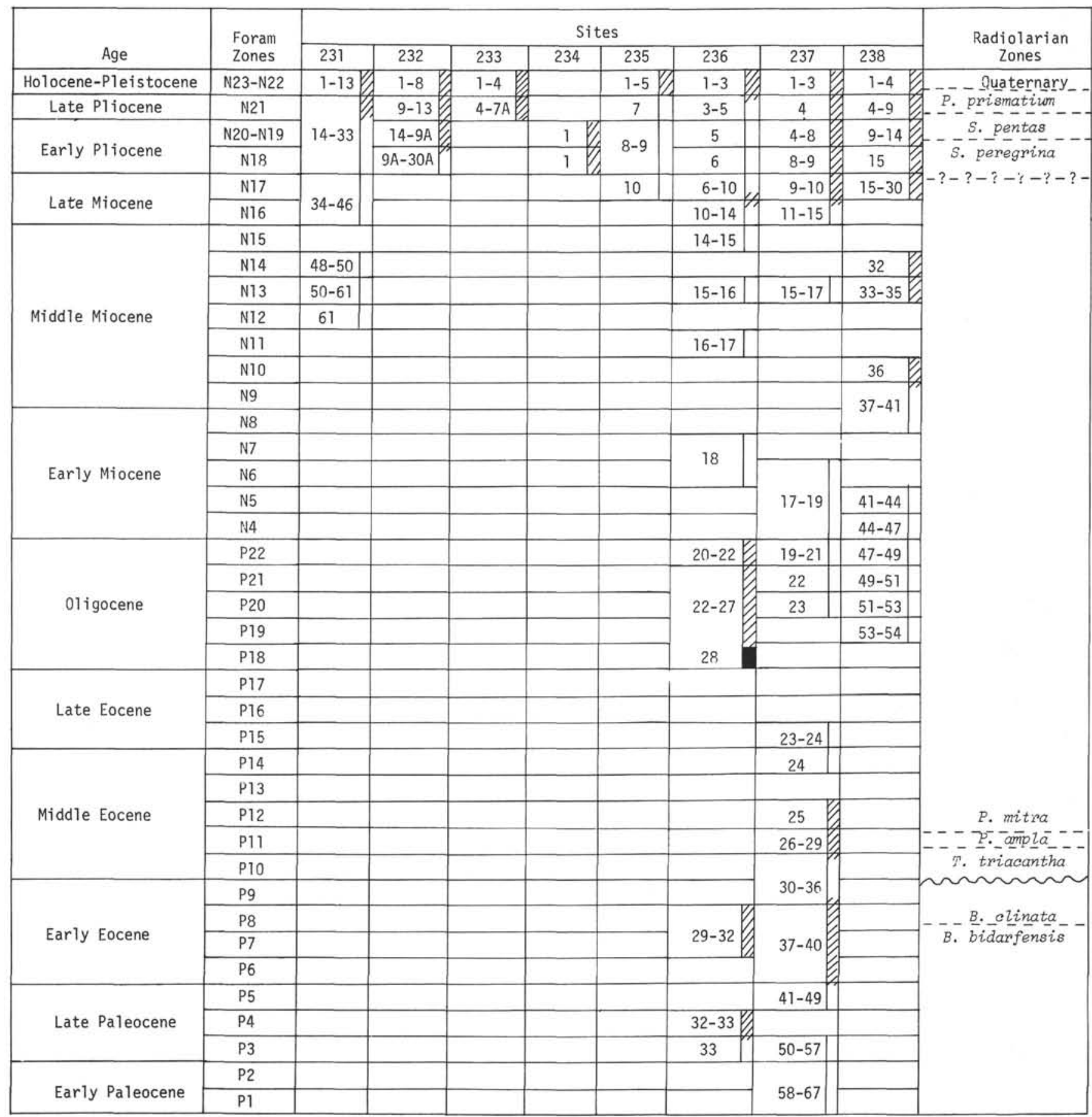

Figure 1. Sediments cored on Leg 24 in the west-central Indian Ocean and Gulf of Aden. Cores are represented by numbered rectangles. Empty rectangles represent calcareous cores lacking siliceous microfossils, hachuring indicates siliceous microfossils occurring in calcareous sediment, and filled rectangles represent cores containing only siliceous microfossils. The radiolarian zonal boundaries indicated in the right-hand column are located only approximately. For more precise information see Tables 1-9. 
TABLE 1

Radiolarians from Site 232 (Hole 232)

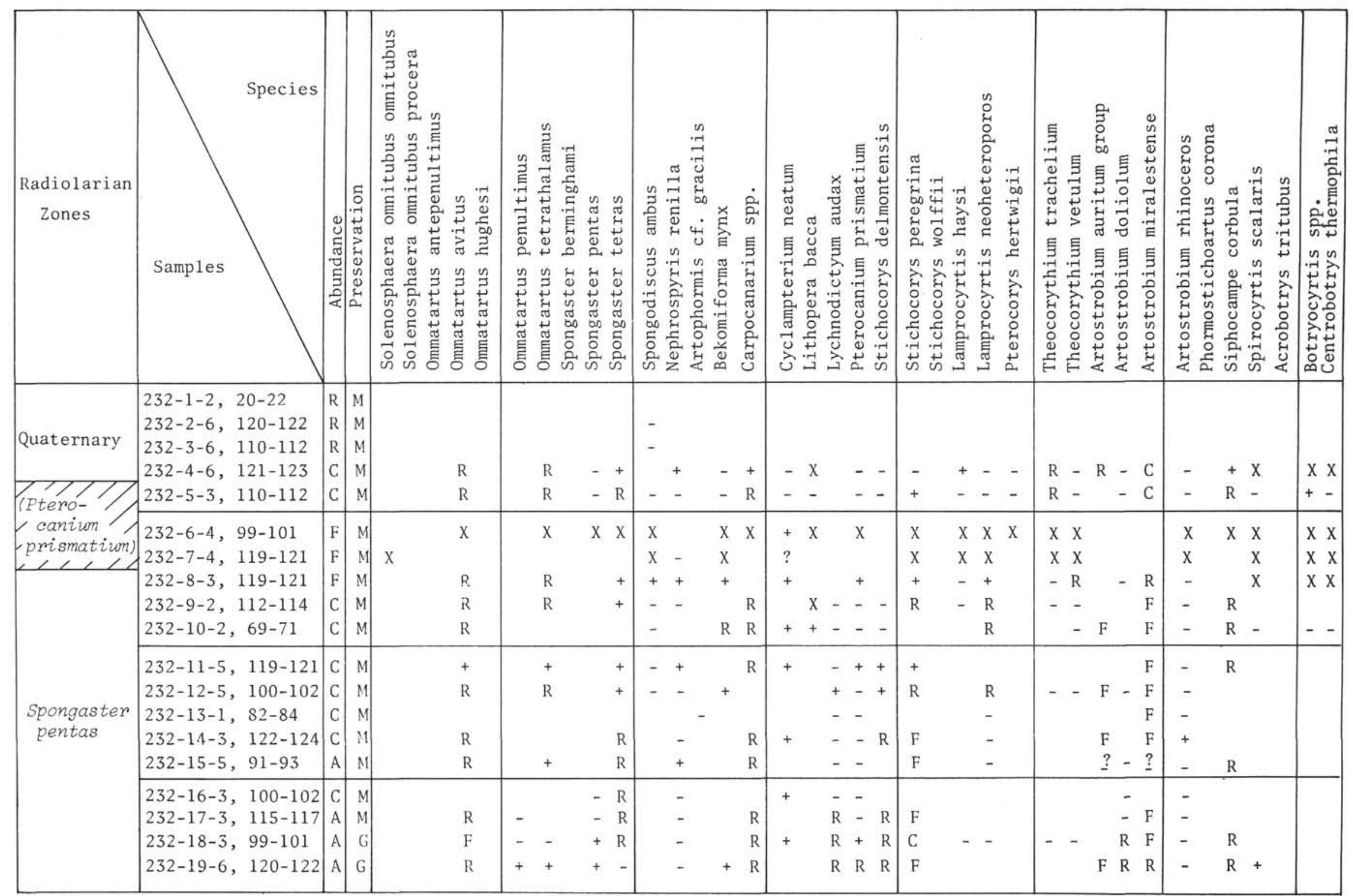


TABLE 1 - Continued

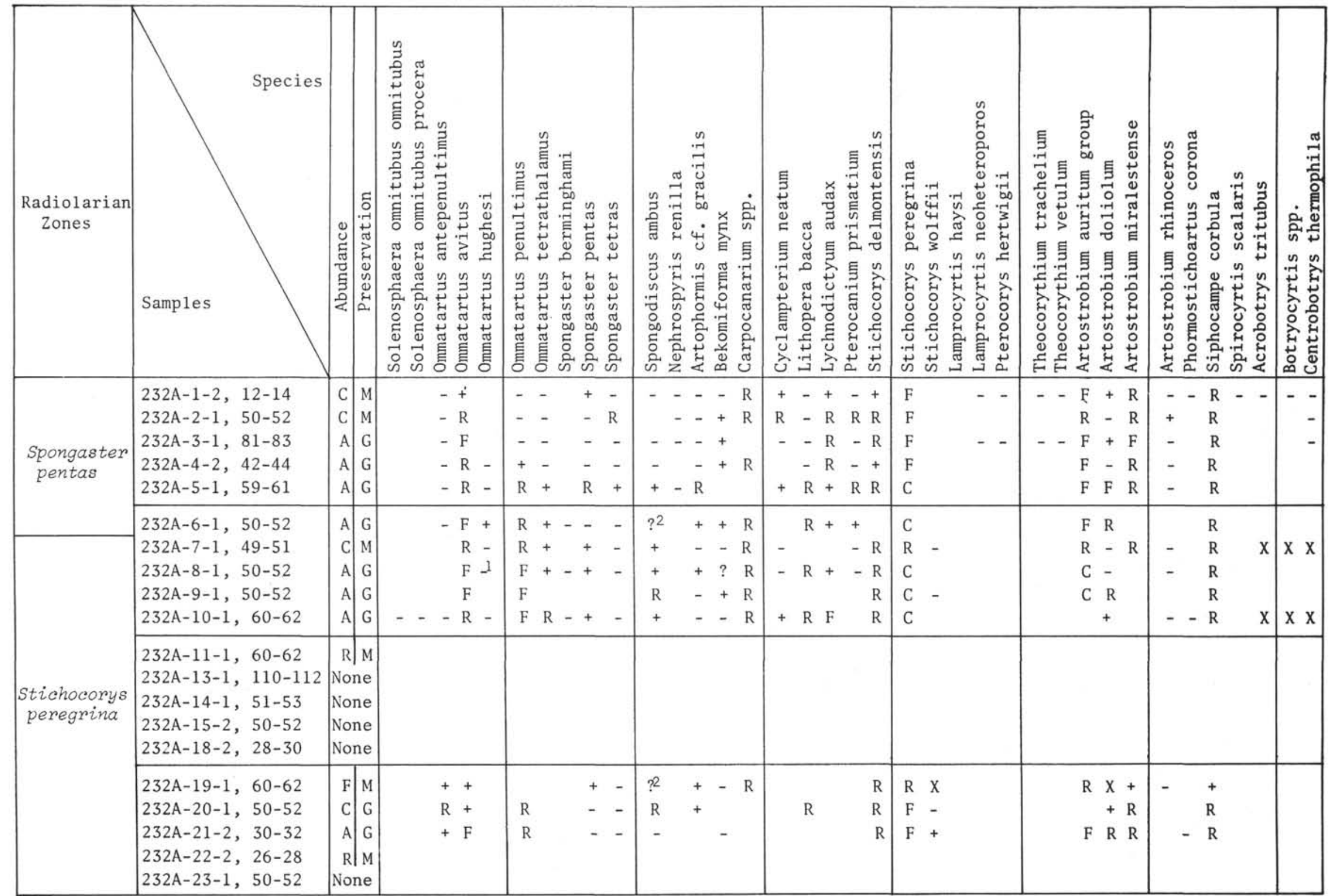

${ }^{1}$ Although Ommatartus hughesi is absent here, there are rare specimens of an artiscin with multiple polar caps which we identify as Ommatartus didymus (Ehrenberg) (Sanfilippo and Riedel in Sanfilippo et al., in press).

${ }^{2}$ Fragments only, including the central portion and some outer meshwork extending to a part of the margin. 
TABLE 2

Radiolarians from Site 233 (Hole 233)

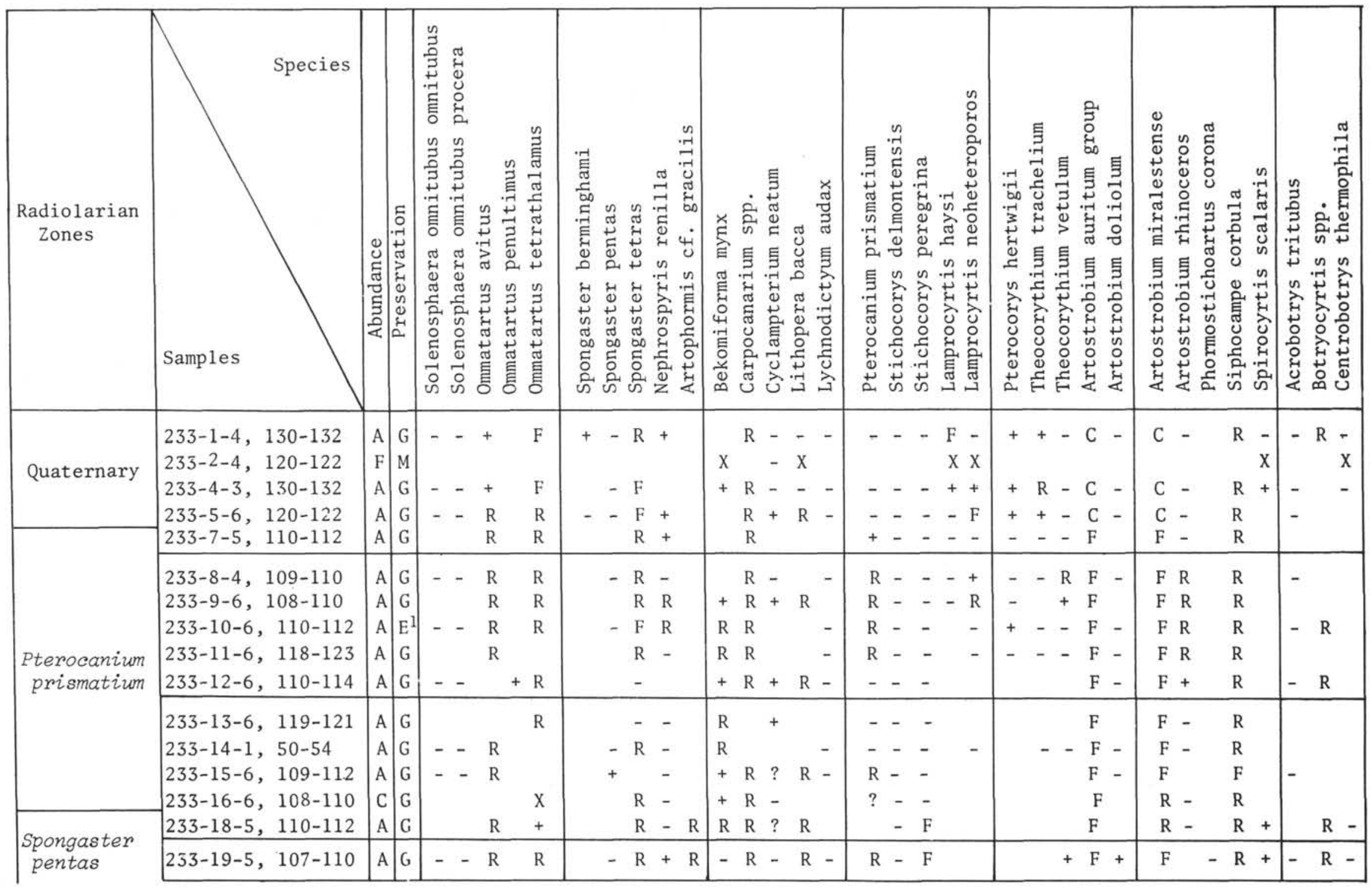

1 Some of the radiolarians in this assemblage are pyritized. 
TABLE 2 - Continued

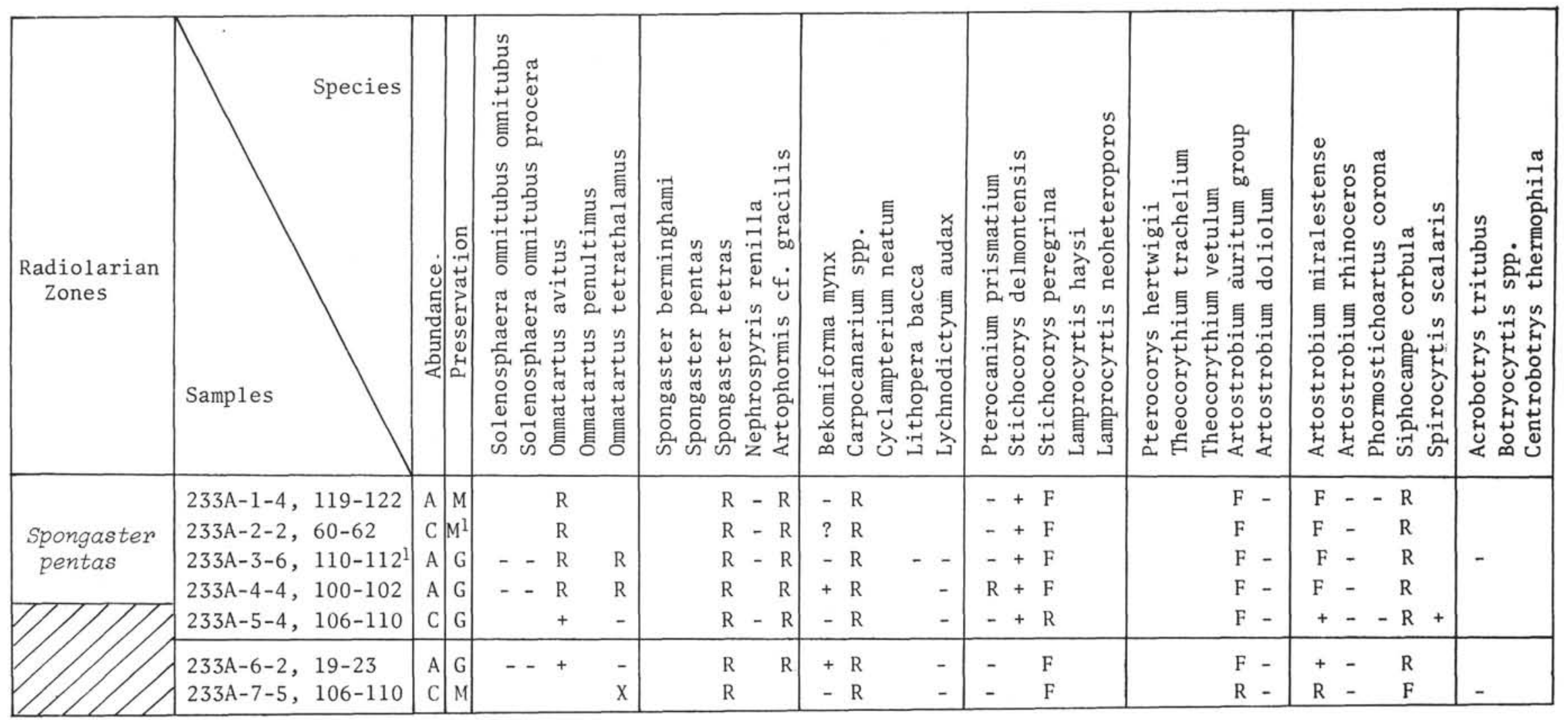

${ }^{1}$ Some of the radiolarians in this assemblage are pyritized. 
TABLE 3

Radiolarians from Site 235

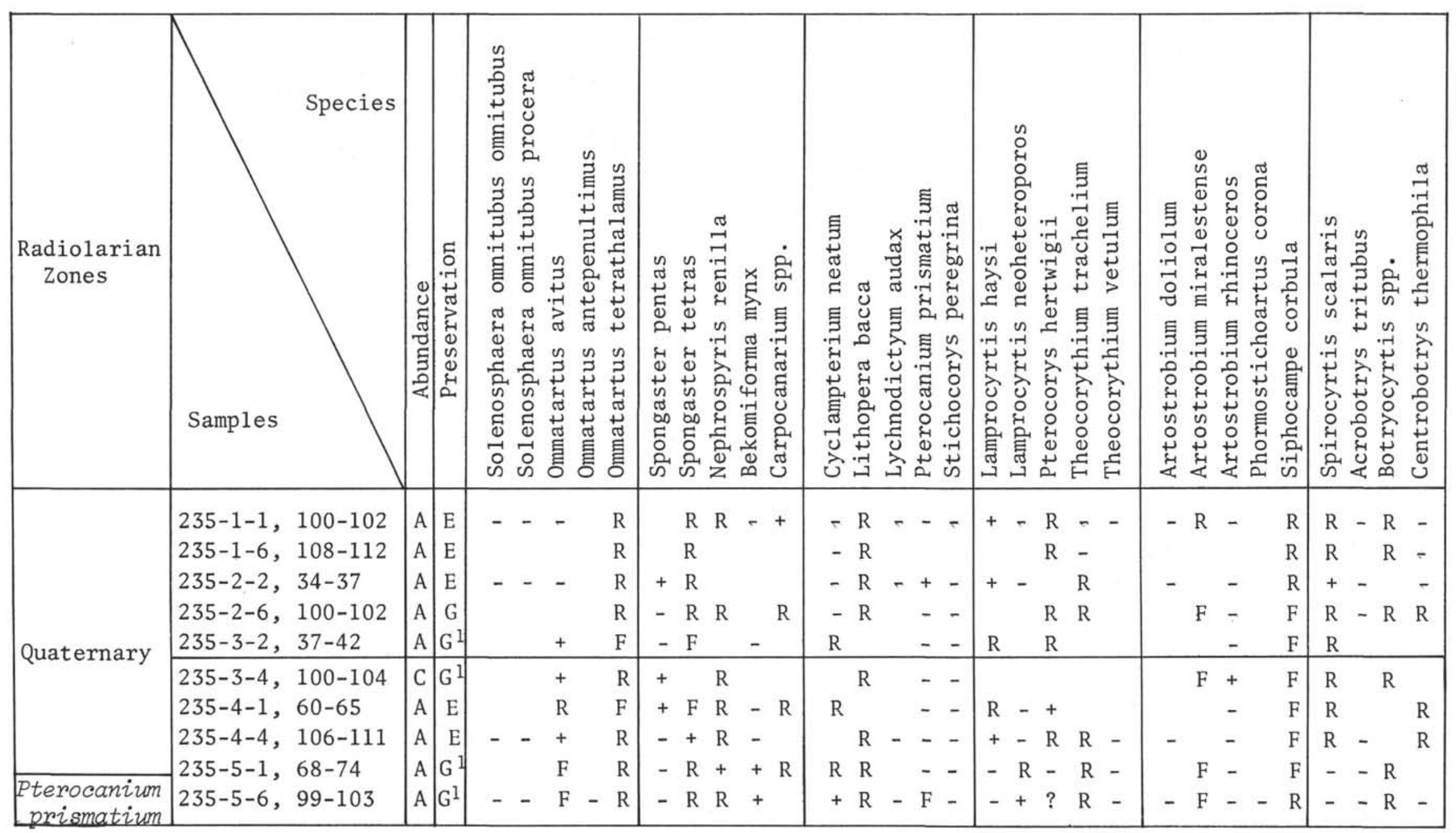

${ }^{1}$ Rare specimens are partially pyritized. 
TABLE 4

Radiolarians from Site 236

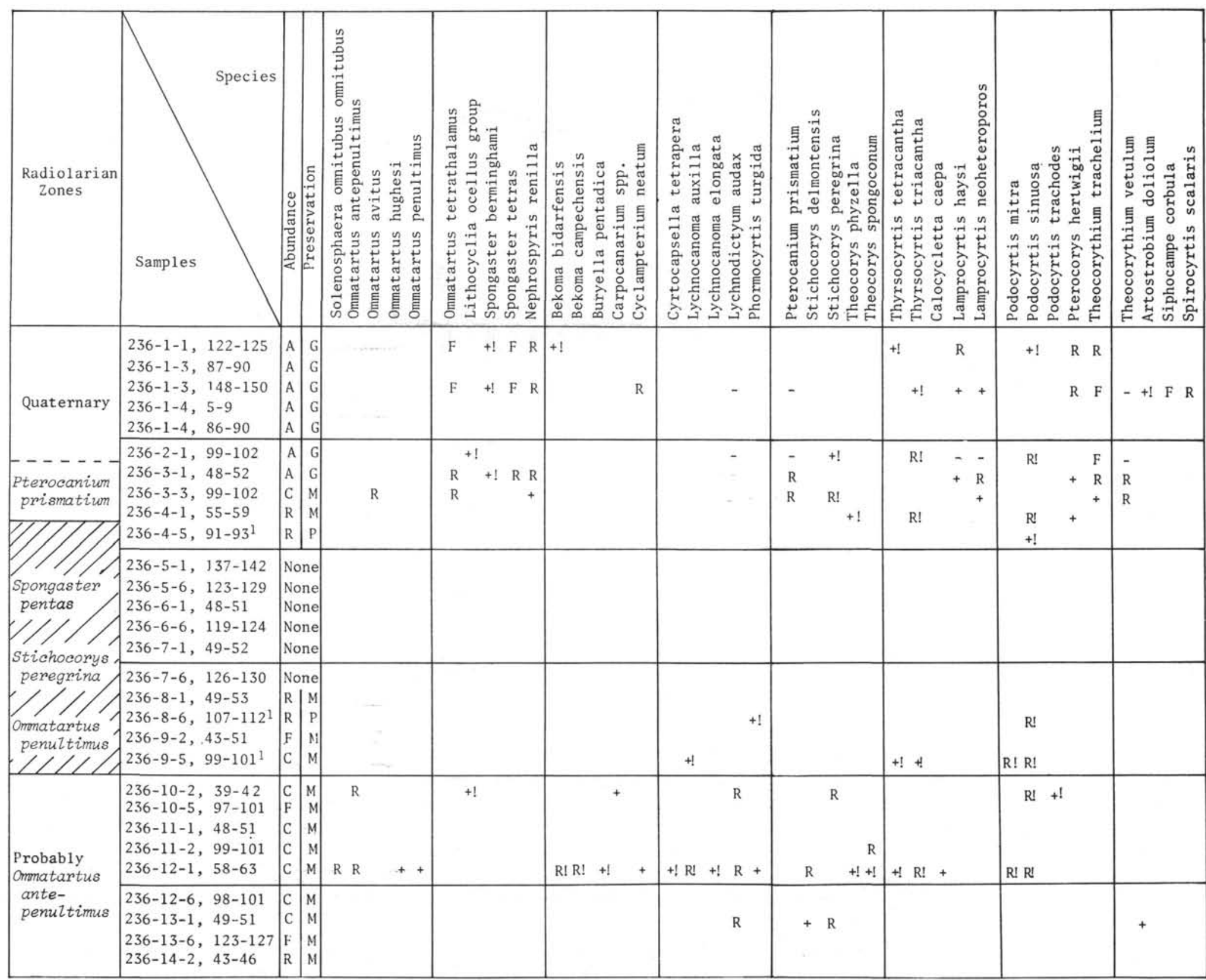

${ }^{1}$ In these assemblages, all of the recognized radiolarians are believed to be reworked, and none autochthonous. 
TABLE 5

Radiolarians from Site 236 (Second Half)

\begin{tabular}{|c|c|c|c|c|c|c|c|c|c|c|}
\hline $\begin{array}{l}\text { Radiolarian } \\
\text { zones }\end{array}$ & Samples & 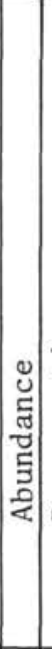 & & 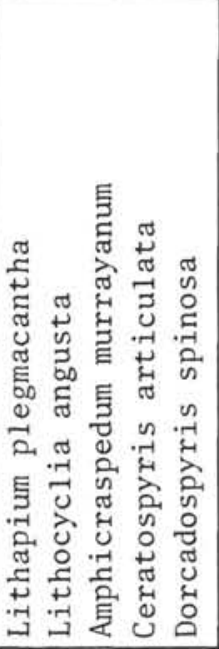 & 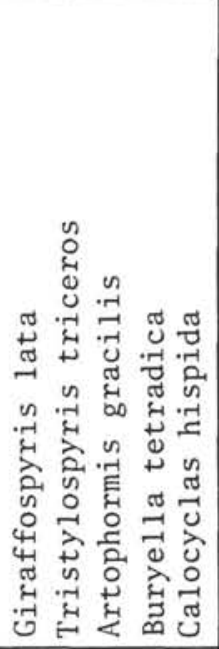 & 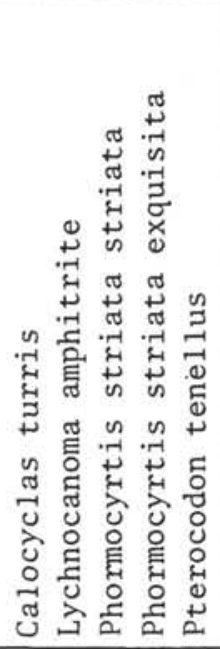 & 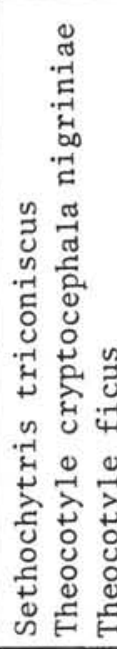 & 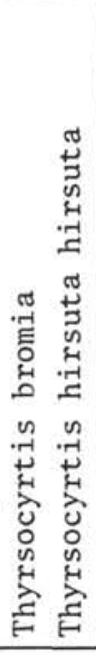 & 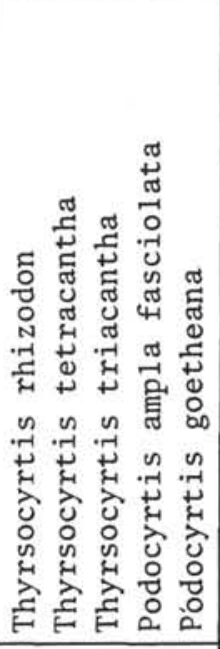 & 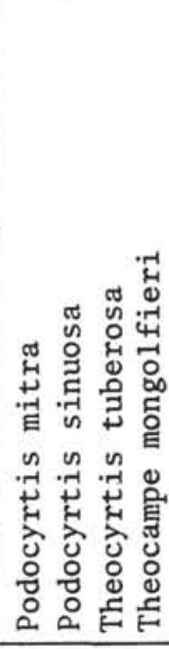 \\
\hline \multirow{4}{*}{$\begin{array}{l}\text { Approxi- } \\
\text { mately } \\
\text { Theocyrtis } \\
\text { tuberosa }\end{array}$} & $\begin{array}{ll}236-20-2, & 42-46 \\
236-20-5, & 50-52 \\
236-20-5, & 98-101 \\
236-21-1, & 47-52 \\
236-21-6, & 99-102\end{array}$ & $\mid \begin{array}{l}F \\
R \\
F \\
F \\
\text { No }\end{array}$ & $\begin{array}{l}M \\
P \\
M \\
M \\
\text { ne }\end{array}$ & $+!$ & $\begin{array}{l}+! \\
\\
\end{array}$ & & & + & $\mathrm{R}$ & \\
\hline & $\begin{array}{ll}236-22-1, & 115-120 \\
236-22-4, & 120-124 \\
236-23-1, & 73-77 \\
236-23-6, & 110-114 \\
236-24-1, & 49-51\end{array}$ & \begin{tabular}{l|}
$\mathrm{C}$ \\
$\mathrm{R}$ \\
$\mathrm{C}$ \\
$\mathrm{C}$ \\
$\mathrm{A}$
\end{tabular} & $\begin{array}{l}M \\
P \\
M \\
M \\
M\end{array}$ & $+!$ & $\begin{array}{l}\mathrm{C} \\
\mathrm{C}+\end{array}$ & + & + & & + & $+!$ \\
\hline & $\begin{array}{ll}236-24-6, & 117-120 \\
236-25-1, & 49-51 \\
236-25-6, & 99-103 \\
236-26-1, & 49-51 \\
236-26-6, & 102-107\end{array}$ & $\mid \begin{array}{l}A \\
A \\
A \\
A \\
A\end{array}$ & $\begin{array}{l}\text { G } \\
\text { G } \\
\text { G } \\
\text { G } \\
\text { G }\end{array}$ & $\begin{array}{l}+ \\
R\end{array}$ & + + F + +! R & $R+R+!+!$ & $+! \mathrm{R} !$ & $\mathrm{R}+!$ & R R R +! + & + \\
\hline & $\begin{array}{ll}236-27-2, & 33-36 \\
236-28-1, & 82-87 \\
236-28-1, & 127-130 \\
236-32-3, & 47-50 \\
236-33-2, & 25-29 \\
236-33-2, & 58-60\end{array}$ & \begin{tabular}{l|}
$\mathrm{F}$ \\
$\mathrm{A}$ \\
$\mathrm{F}$ \\
$\mathrm{F}$ \\
$\mathrm{R}$ \\
$\mathrm{R}$
\end{tabular} & \begin{tabular}{l|}
$M$ \\
$M$ \\
$P$ \\
$P$ \\
$P$ \\
$P$
\end{tabular} & + & $\begin{array}{lll}C & R & R\end{array}$ & $R++$ & & $\mathrm{R}$ & $\mathrm{R} R \mathrm{R} R$ & $\mathrm{R}$ \\
\hline
\end{tabular}

${ }^{1}$ This assemblage (on Slide 1 at B26/4) includes a fragment of the peculiar looped spine recorded from the basal Eocene by Riedel and Sanfilippo (in press, pl. 5 , figs. 8,9 ). 
TABLE 6

Radiolarians from Site 237

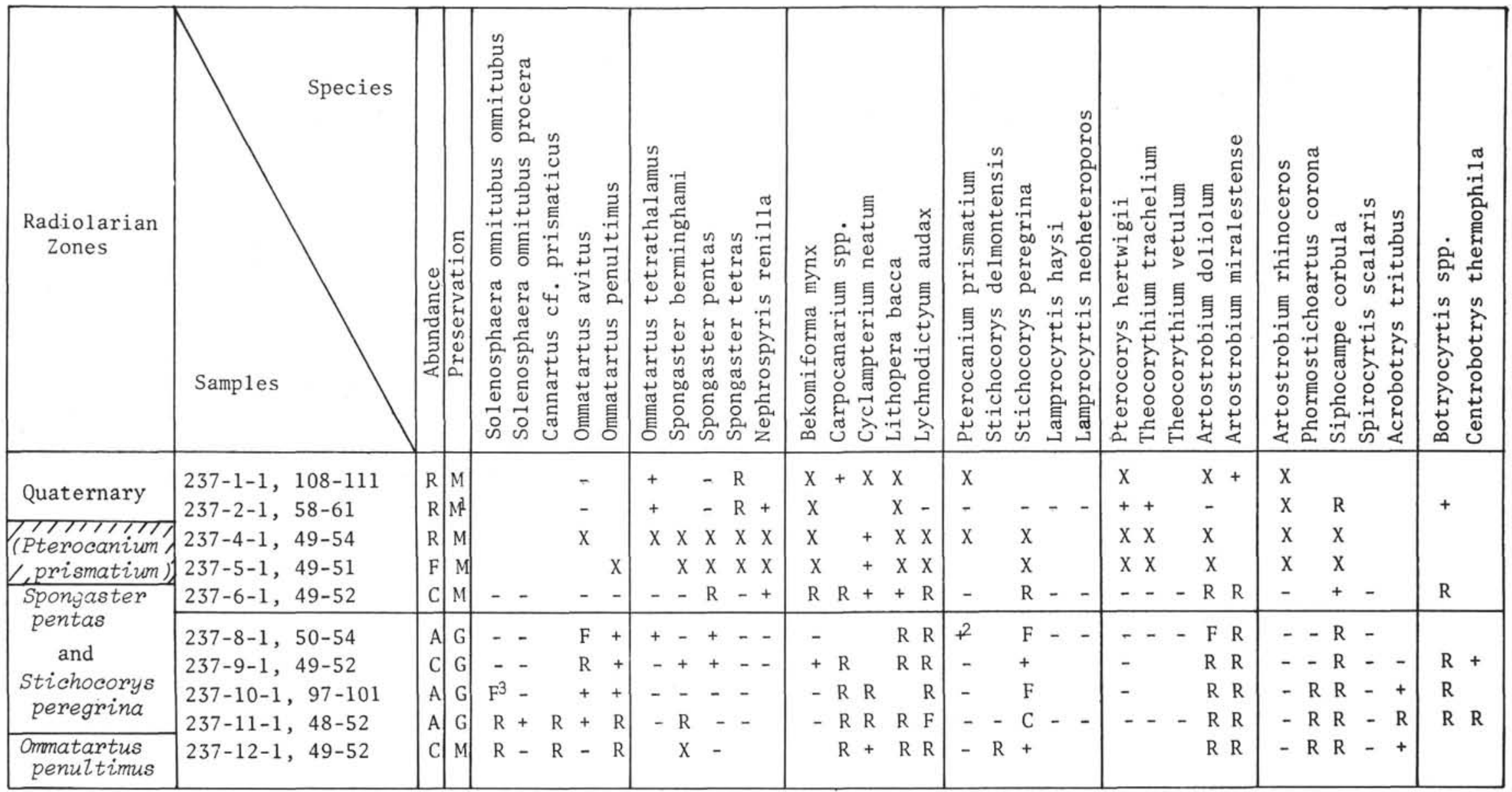

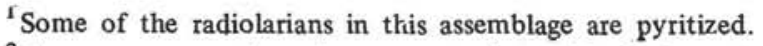

${ }^{2}$ These are apparently early representatives, with the thoracic shoulders only slightly thorny.

${ }^{3}$ These latest specimens of Solenosphaera omnitubus omnitubus are hyaline, with practically no pores between the short, wide tubes. 
TABLE 7

Radiolarians from Site 237

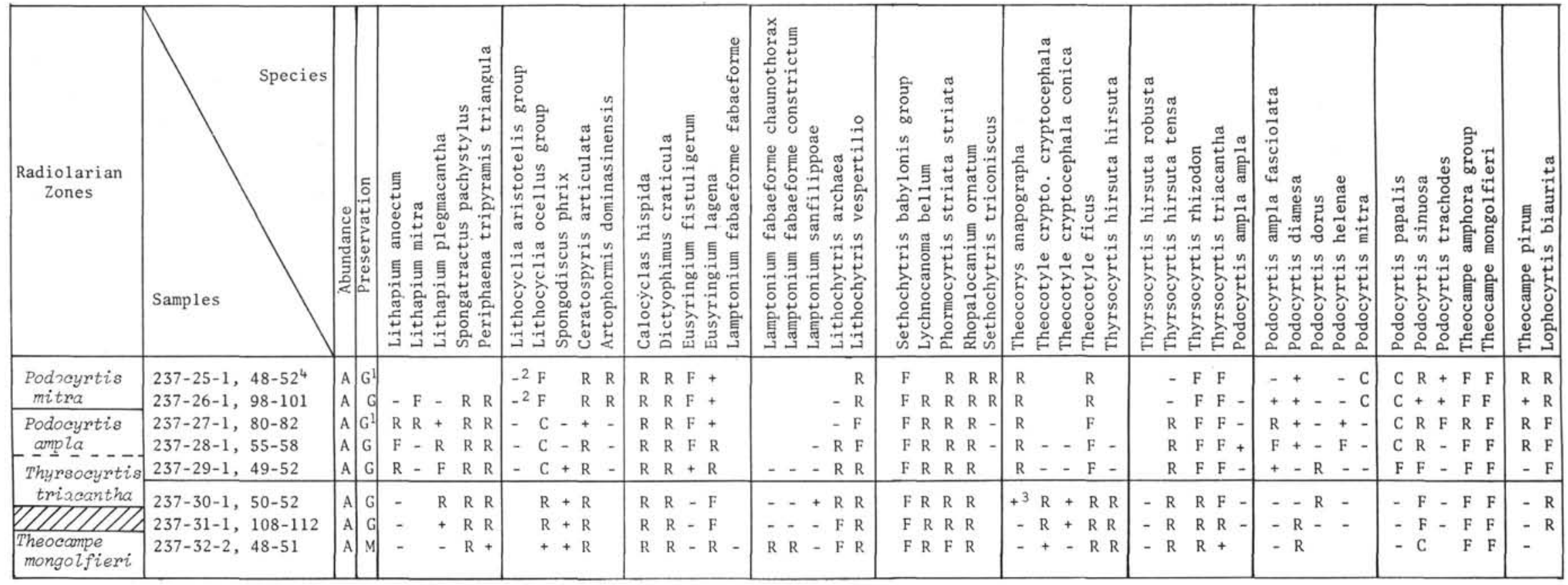

${ }^{1}$ Some of the radiolarians in this assemblage are pyritized.

${ }^{2}$ These samples contain rare specimens of a form showing some superficial resemblance to this group (pl. 1, fig. 11).

${ }^{3}$ In addition to Theocorys anapographa, there are rare specimens of a two-segmented species with rather similar pores (pl. 3, figs. 1-3).

${ }^{4}$ In this assemblage are rare examples of an extraordinary theoperid cephalis with three strong horns almost equally developed (pl. fig. 5,6 ). We have not found any more complete skeleton. 
TABLE 8

Radiolarians from Site 237

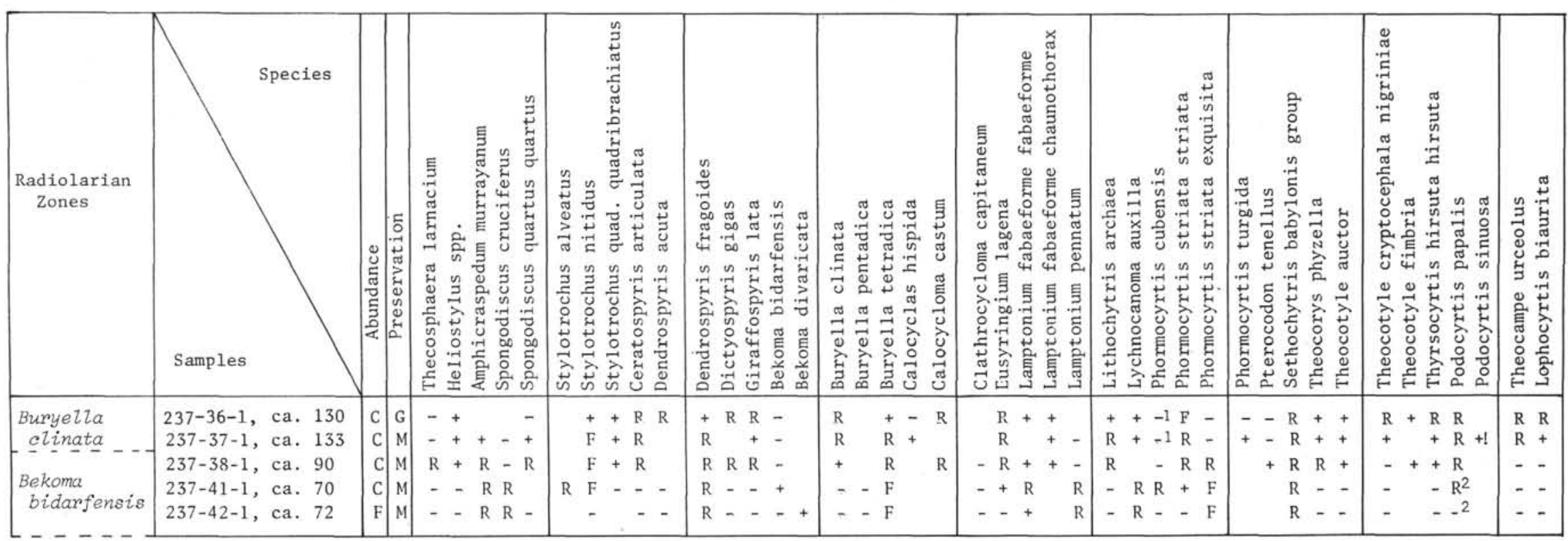

${ }^{1}$ These assemblages contain specimens of Tricolocampe vitrea Krasheninnikov (pl. 3, fig. 4) - rare in the sample from Core 37 and very rare in the sample from Core 36.

${ }^{2}$ The feet and distal part of the abdomen are often corroded. 
TABLE 9

Radiolarians from Site 238

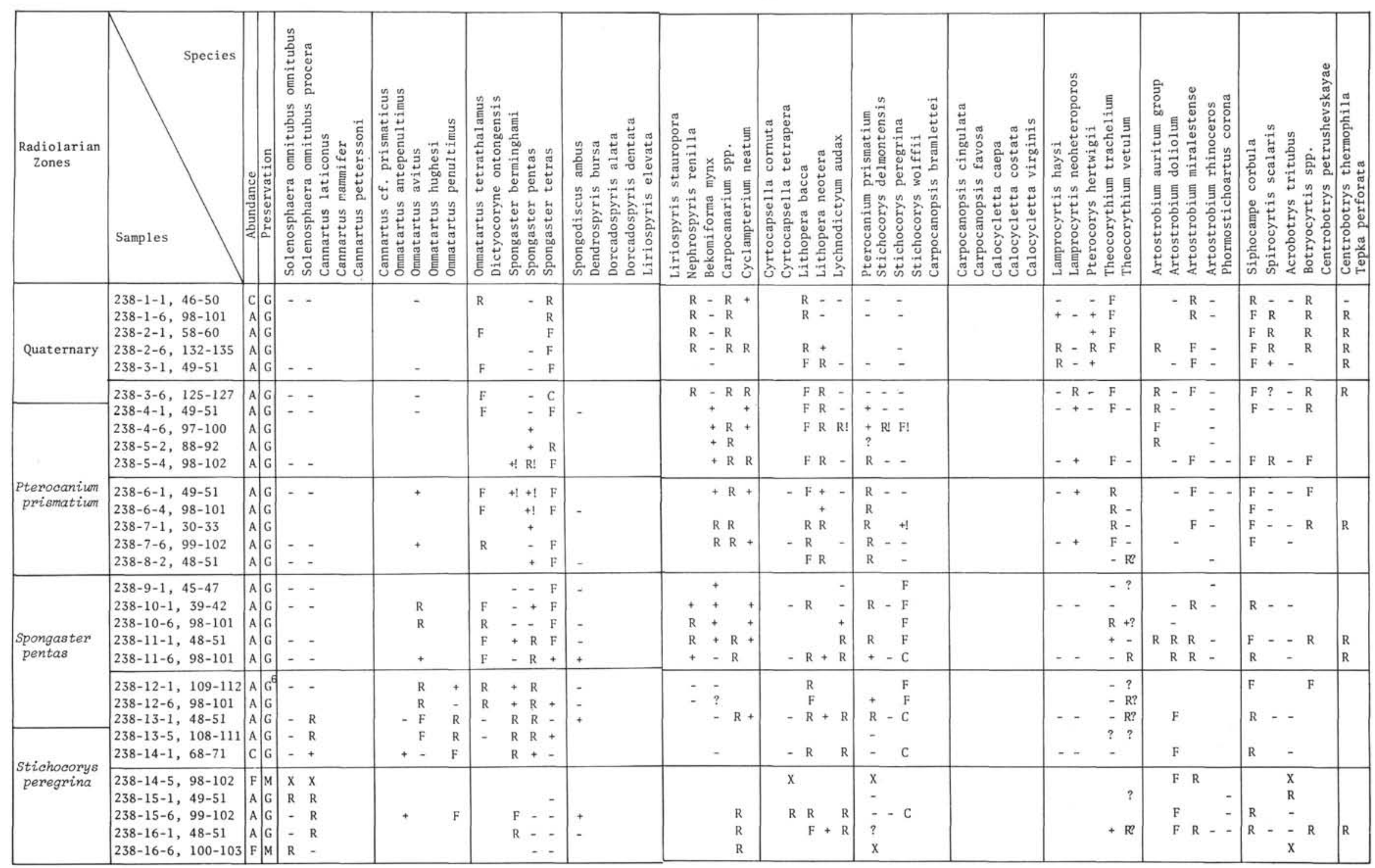


TABLE 9 - Continued

\begin{tabular}{|c|c|c|c|c|c|c|c|c|c|c|c|c|c|c|c|}
\hline $\begin{array}{l}\text { Radiolarian } \\
\text { Zones }\end{array}$ & Samples & & 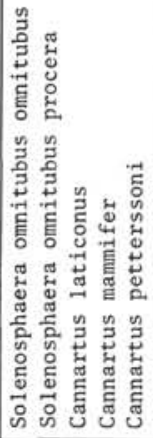 & 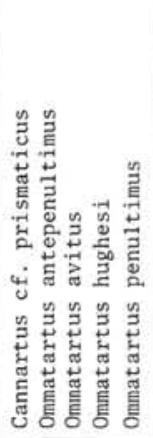 & 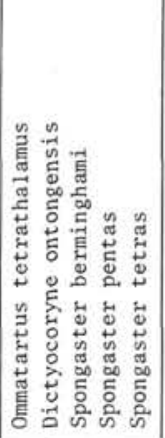 & 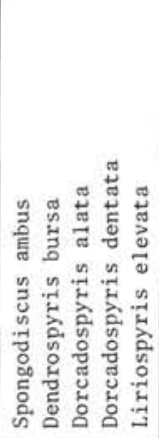 & 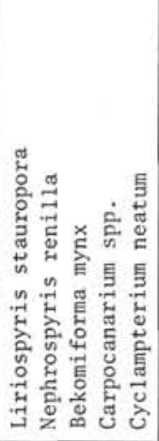 & 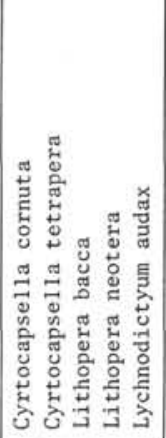 & 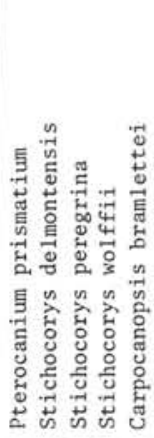 & & 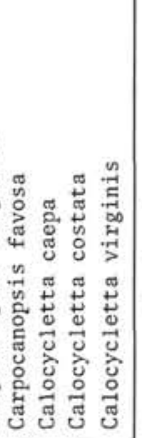 & 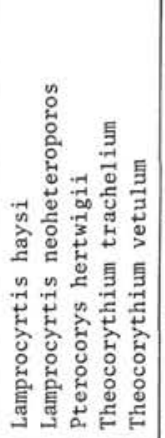 & 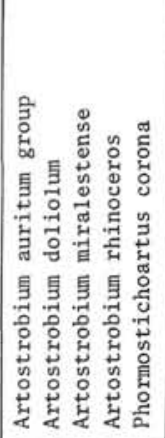 & 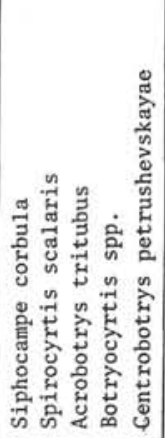 & 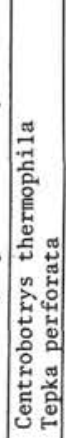 \\
\hline \multirow{3}{*}{$\begin{array}{l}\text { Comnartus } \\
\text { petterssoni } \\
\text { and } \\
\text { Dorcado- } \\
\text { spyris } \\
\text { alata } \\
\\
\begin{array}{c}\text { Calocycletta } \\
\text { costata }\end{array}\end{array}$} & 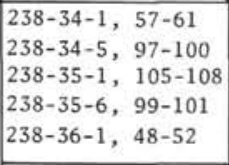 & \begin{tabular}{r|l|l} 
F & $M$ \\
C & $M$ \\
None \\
F & $M$ \\
$C$ & $M$
\end{tabular} & $\begin{array}{l}x+R^{+} \\
x=- \\
x=-\end{array}$ & \multicolumn{2}{|c|}{\begin{tabular}{|l|l|}
+ & \\
- &
\end{tabular}} & $\begin{array}{r}+ \\
+R^{1} \\
R^{1} \\
R^{1}\end{array}$ & & $\begin{array}{lll}- & + & - \\
- & - \\
- & -\end{array}$ & $\begin{array}{cccc} & + & + \\
R & R & R \\
X & \\
- & \\
& & R\end{array}$ & \multicolumn{2}{|r|}{$\begin{array}{ll} & + \\
x & \bar{x}\end{array}$} & \multicolumn{2}{|r|}{$\begin{array}{ll}- & \\
++ & + \\
- & x\end{array}$} & - $\quad R$ & \\
\hline & $\begin{array}{l}238-36-6,98-101 \\
238-37-1,59-61 \\
238-37-6,48-54 \\
238-38-6,48-51 \\
238-38-6,98-101 \\
\end{array}$ & $\begin{array}{rl}R & P \\
C & M \\
\text { None } \\
\text { N } \\
\text { C } M \\
\text { A } M\end{array}$ & & & & $\begin{array}{l}+1+ \\
+1+ \\
+1 \\
+1\end{array}$ & & & $\mathrm{~F}$ & & $\begin{array}{l}x \times \\
++\end{array}$ & & & & \\
\hline & $238-39-2,45-48$ & $A \mid M$ & & & & $\mathrm{R}^{1}$ & & $\mathrm{R}$ & $\mathrm{F}$ & & $\mathrm{R}$ & & & & \\
\hline
\end{tabular}

${ }^{1}$ These records of occurrence are based on isolated feet only.

${ }^{2}$ These well-preserved specimens retain the terminal lamellar feet (pl. 3, fig. 7).

${ }^{3}$ This assemblage includes rare specimens of the rather similar form with protuberances on the cortical shell (pl. 1, fig. 9).

${ }^{4}$ Above the range of the form that we have recorded as Cannartus sp. cf. C. prismaticus occurs a rather similar form but with a distinct equatorial constriction (pl. 1, fig. 8 ).

${ }^{5}$ These specimens, intermediate between Cyclampterium (?) brachythorax and $C$. (?) neatum, are morphologically similar to $C$. (?) tanythorax.

${ }^{6}$ Some specimens in these assemblages are pyritized. 
TABLE 10

Radiolarians from Site 73

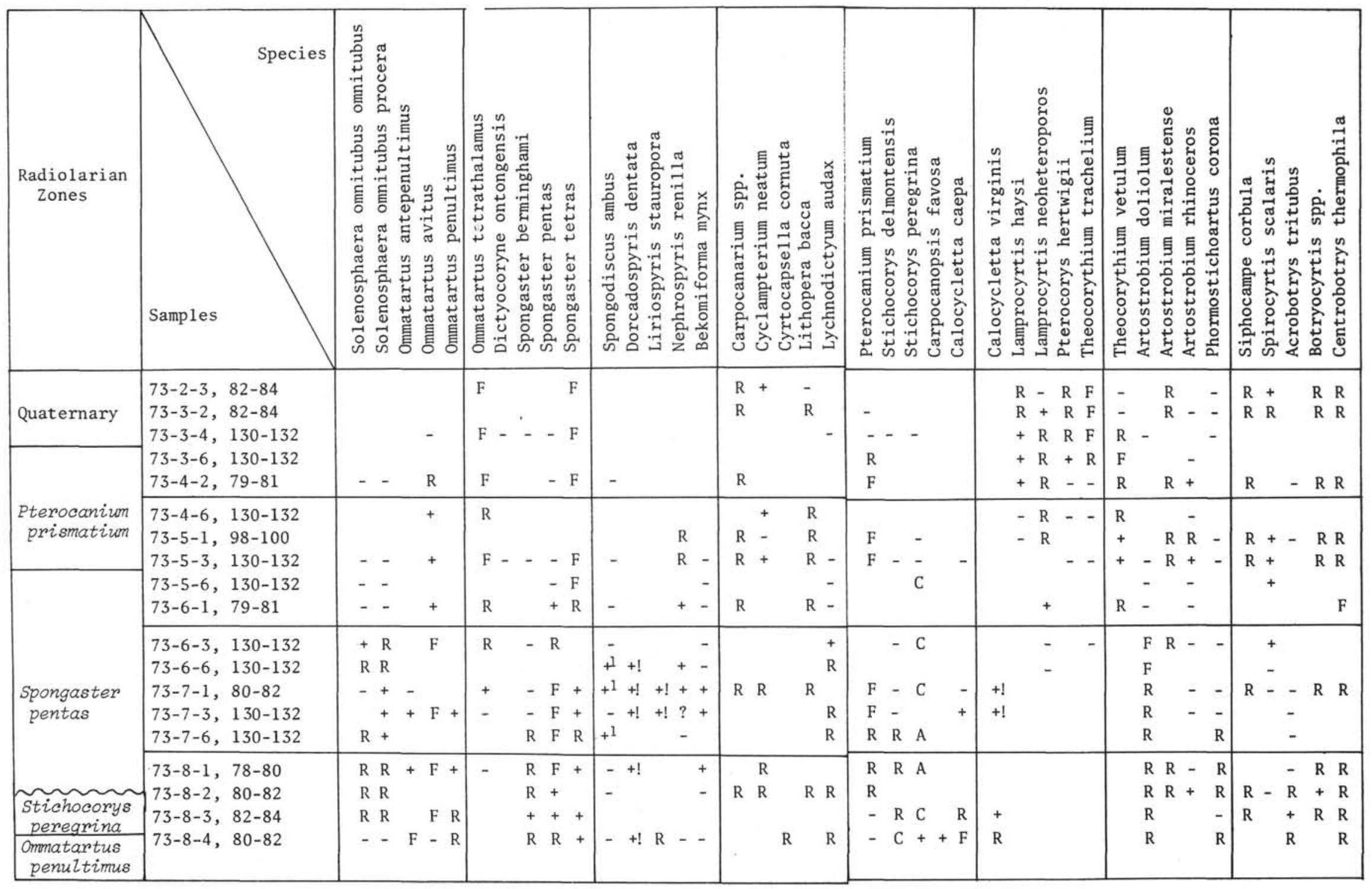

${ }^{1}$ Fragments of Spongodiscus ambus are recorded here, but only when they include both the central structure and a part of the margin. 
TABLE 11

Radiolarians from Hole 77B

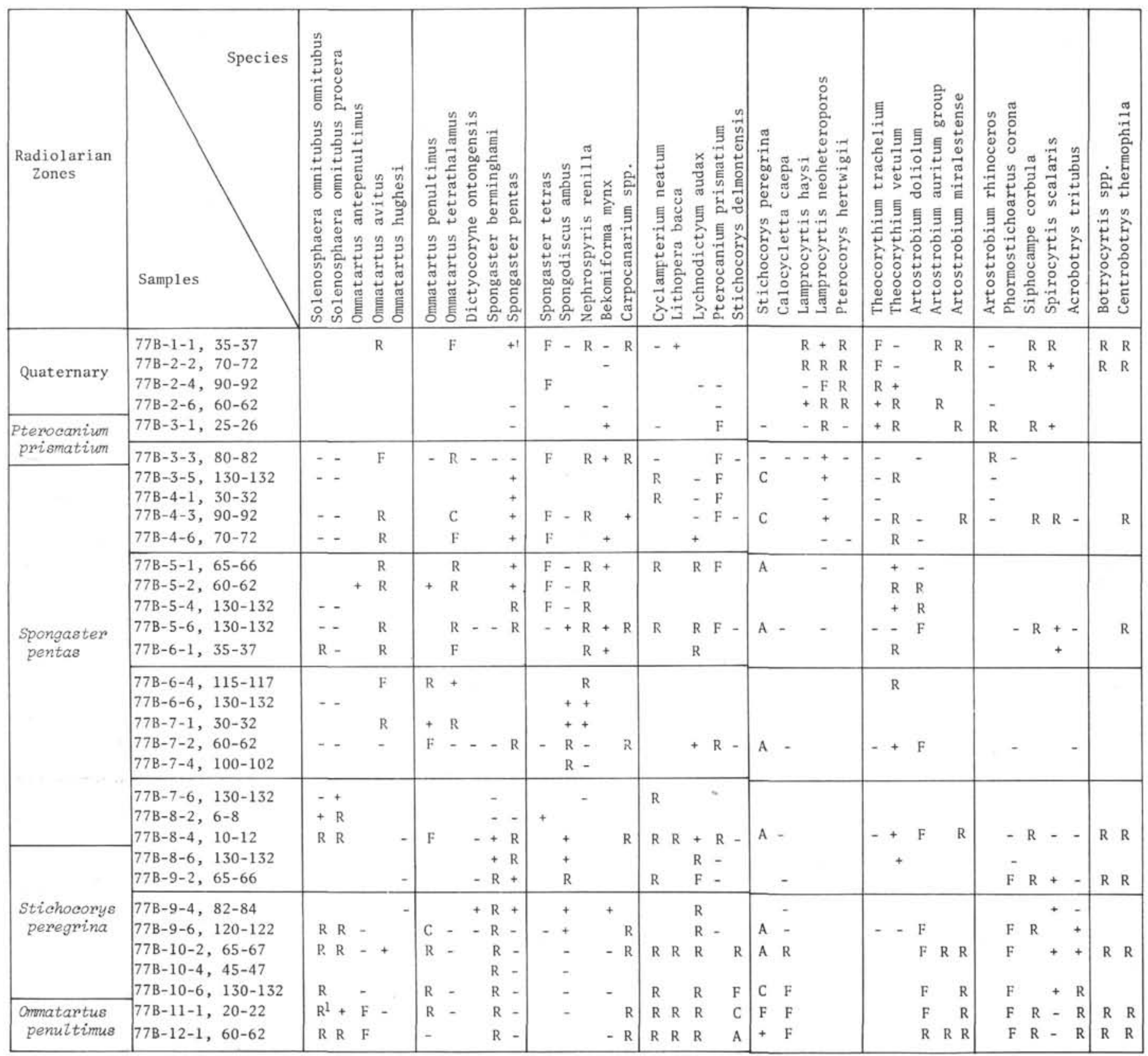

${ }^{1}$ These specimens have hyaline shells, with practically no pores between the short, wide tubes. 
TABLE 12

Late Neogene Radiolarian Events in the Indian Ocean (Sites 232-238) and Tropical Pacific (Sites 73, 77)

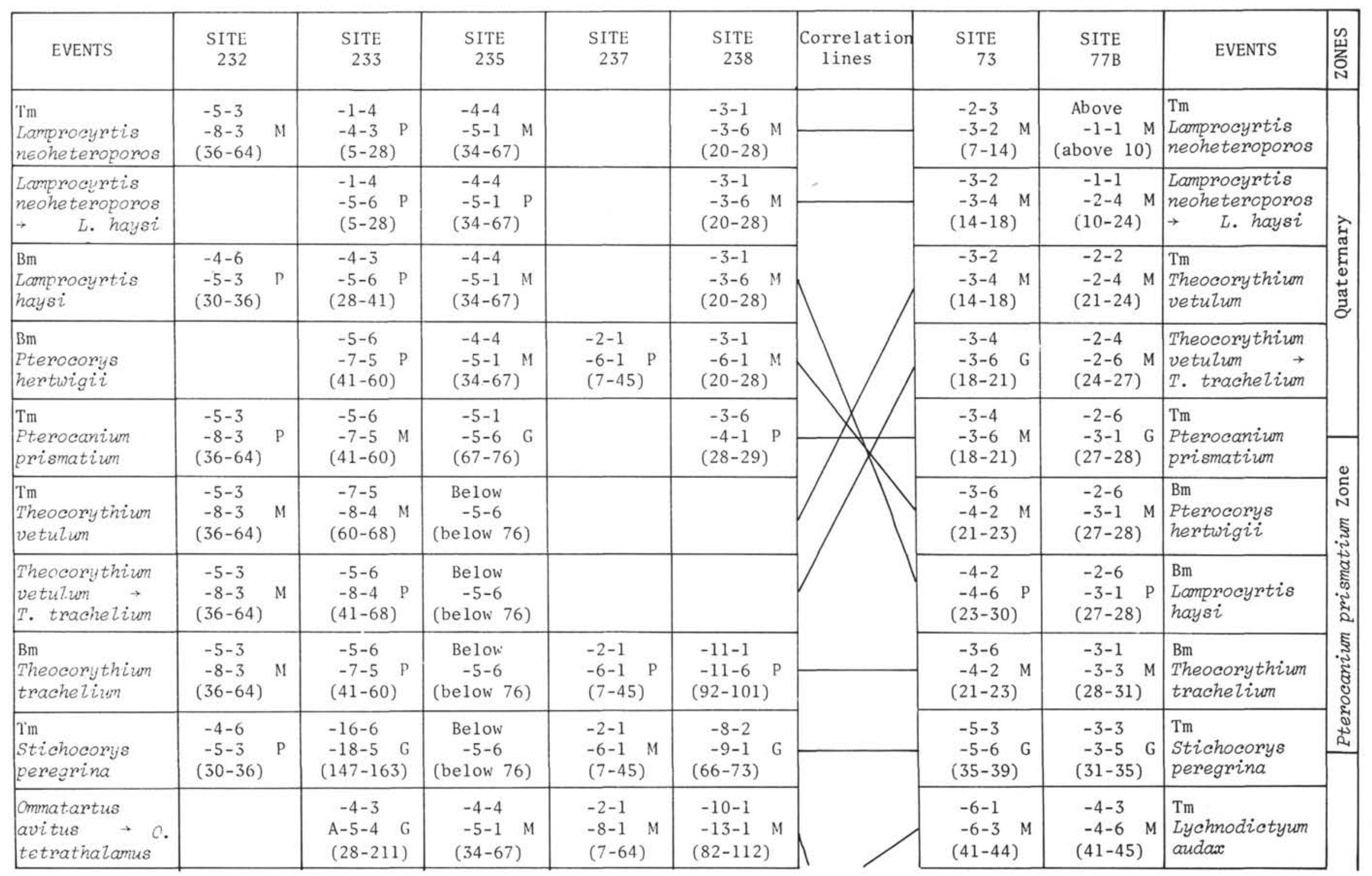


TABLE 12 - Continued

\begin{tabular}{|c|c|c|c|c|c|c|c|c|c|}
\hline EVENTS & $\begin{array}{r}\text { SITE } \\
232\end{array}$ & $\begin{array}{r}\text { SITE } \\
233\end{array}$ & $\begin{array}{r}\text { SITE } \\
235\end{array}$ & $\begin{array}{r}\text { SITE } \\
237\end{array}$ & $\begin{array}{r}\text { SITE } \\
238\end{array}$ & $\begin{array}{l}\text { Correlation } \\
\text { lines }\end{array}$ & $\begin{array}{l}\text { SITE } \\
73\end{array}$ & $\begin{array}{r}\text { SITE } \\
77 \mathrm{~B}\end{array}$ & EVENTS \\
\hline $\begin{array}{l}\text { Tm } \\
\text { Lychnodictyum } \\
\text { audax }\end{array}$ & $\begin{array}{l}-16-3 \\
-17-3 \quad M \\
(140-150)\end{array}$ & $\begin{array}{c}\text { Below } \\
\text { A-7-5 } \\
\text { (below 232) }\end{array}$ & $\begin{array}{c}\text { Below } \\
-5-6 \\
\text { (below 76) }\end{array}$ & $\begin{array}{l}-2-1 \\
-6-1 \quad M \\
(7-45)\end{array}$ & $\begin{array}{c}-10-1 \\
-10-6 \\
(82-91)\end{array}$ & & $\begin{array}{l}-5-6 \\
-6-1 \quad M \\
(39-41)\end{array}$ & $\begin{array}{l}-4-3 \\
-5-1 \quad P \\
(41-46)\end{array}$ & $\begin{array}{l}\mathrm{Tm} \\
\text { Spongaster } \\
\text { pentas }\end{array}$ \\
\hline $\begin{array}{l}\text { Tm } \\
\text { Spongaster } \\
\text { pentas }\end{array}$ & $\begin{array}{l}-17-3 \\
-18-3 \quad P \\
(150-159)\end{array}$ & & $\begin{array}{l}\text { Probab1y } \\
\text { below }-5-6 \\
\text { (below } 76 \text { ) }\end{array}$ & $\begin{array}{l}-2-1 \\
-6-1 \quad M \\
(7-45)\end{array}$ & $\begin{array}{c}-4-1 \\
-4-6 \quad P \\
(29-34)\end{array}$ & & $\begin{array}{l}-6-1 \\
-6-3 \quad G \\
(41-44)\end{array}$ & $\begin{array}{l}-5-1 \\
-5-2 \quad M \\
(46-48)\end{array}$ & $\begin{array}{l}\text { Tm } \\
\text { Artostrobium } \\
\text { doliolum }\end{array}$ \\
\hline $\begin{array}{l}\text { Tm } \\
\text { Artostrobium } \\
\text { doliolum }\end{array}$ & $\begin{array}{c}-17-3 \\
-18-3 \\
(150-159)\end{array}$ & $\begin{array}{c}\text { Below } \\
\text { A-7-5 } \\
\text { (below 232) }\end{array}$ & $\begin{array}{c}\text { Below } \\
-5-6 \\
\text { (below 76) }\end{array}$ & $\begin{array}{l}-2-1 \\
-6-1 \quad M \\
(7-45)\end{array}$ & $\begin{array}{l}-10-6 \\
-11-1 \quad M \\
(91-92)\end{array}$ & & $\begin{array}{l}-6-1 \\
-8-4 \quad M \\
(41-63)\end{array}$ & $\begin{array}{l}-5-4 \\
-5-6 \\
(52-54)\end{array}$ & $\begin{array}{l}\text { Spongaster } \\
\text { pentas } \\
\rightarrow \quad \text { S. tetras }\end{array}$ \\
\hline $\begin{array}{l}\text { Spongaster } \\
\text { pentas } \rightarrow \\
S \text {. tetras }\end{array} \rightarrow$ & & & $\begin{array}{c}\text { Below } \\
-5-6 \\
\text { (below 76) }\end{array}$ & $\begin{array}{l}-2-1 \\
-6-1 \quad M \\
(7-45)\end{array}$ & $\begin{array}{l}-11-1 \\
-11-6 \\
(92-101)\end{array}$ & & $\begin{array}{c}\text { Below } \\
-8-4 \\
\text { (below 63) }\end{array}$ & $\begin{array}{l}-5-4 \\
-5-6 \\
(52-54)\end{array}$ & $\begin{array}{l}\text { Bm } \\
\text { Spongaster } \\
\text { tetras }\end{array}$ \\
\hline $\begin{array}{l}\text { Bm } \\
\text { Spongaster } \\
\text { tetras }\end{array}$ & $\begin{array}{l}A-5-1 \\
A-6-1 \quad P \\
(198-207)\end{array}$ & $\begin{array}{c}\text { Below } \\
\text { A-7-5 } \\
\text { (below 232) }\end{array}$ & $\begin{array}{c}\text { Below } \\
-5-6 \\
\text { (below 76) }\end{array}$ & $\begin{array}{l}-2-1 \\
-6-1 \quad M \\
(7-45)\end{array}$ & $\begin{array}{c}-13-5 \\
-14-1 \\
(117-121)\end{array}$ & & $\begin{array}{l}-6-1 \\
-6-3 \quad M \\
(41-44)\end{array}$ & $\begin{array}{l}-5-6 \\
-7-6 \quad P \\
(54-73)\end{array}$ & $\begin{array}{l}\text { Tm Soleno- } \\
\text { sphaera omni- } \\
\text { tubus procera }\end{array}$ \\
\hline $\begin{array}{l}\mathrm{Bm} \\
\text { Nephrospyris } \\
\text { renizla }\end{array}$ & & $\begin{array}{l}-10-6 \\
-11-6 \quad M \\
(90-100)\end{array}$ & $\begin{array}{c}\text { Below } \\
-5-6 \\
\text { (below 76) }\end{array}$ & $\begin{array}{l}-6-1 \\
-8-1 \quad P \\
(45-64)\end{array}$ & $\begin{array}{c}-11-6 \\
-12-1 \\
(101-102)\end{array}$ & & $\begin{array}{l}-6-3 \\
-6-6 \quad P \\
(44-49)\end{array}$ & $\begin{array}{l}-6-1 \\
-6-4 \\
(55-61)\end{array}$ & $\begin{array}{l}\text { Ommatartus } \\
\text { avitus } \rightarrow 0 . \\
\text { tetrathalamus }\end{array}$ \\
\hline $\begin{array}{l}\text { Tm } \\
\text { Spongaster } \\
\text { berminghami }\end{array}$ & & & & $\begin{array}{l}-8-1 \\
-9-1 \quad P \\
(64-74)\end{array}$ & $\begin{array}{c}-10-6 \\
-11-1 \\
(91-92)\end{array}$ & & $\begin{array}{l}-7-3 \\
-7-6 \quad p \\
(54-58)\end{array}$ & $\begin{array}{l}-7-1 \\
-7-2 \quad M \\
(64-66)\end{array}$ & $\begin{array}{l}\text { Bm } \\
\text { Nephrospyris } \\
\text { renizza }\end{array}$ \\
\hline $\begin{array}{l}\text { Tm Soleno- } \\
\text { sphaera omni- } \\
\text { tubus procera }\end{array}$ & & $\begin{array}{c}\text { Below } \\
\text { A-7-5 } \\
\text { (below 232) }\end{array}$ & $\begin{array}{c}\text { Below } \\
-5-6 \\
\text { (below 76) }\end{array}$ & $\begin{array}{l}-10-1 \\
-11-1 \quad P \\
(84-93)\end{array}$ & $\begin{array}{c}-13-1 \\
-13-5 \\
(112-117)\end{array}$ & & $\begin{array}{l}-\varepsilon-3 \\
-8-4 \quad G \\
(62-63)\end{array}$ & $\begin{array}{l}-7-1 \\
-7-2 \quad G \\
(64-66)\end{array}$ & $\begin{array}{l}\text { Ommatartus } \\
\text { penultimus } \\
\text { o. avitus }\end{array}$ \\
\hline $\begin{array}{l}\text { Spongaster } \\
\text { berminghami } \\
\rightarrow \quad S \text {. pentas }\end{array}$ & & & & $\begin{array}{l}-8-1 \\
-11-1 \quad P \\
(64-93)\end{array}$ & $\begin{array}{c}-12-6 \\
-14-1 \\
(110-121)\end{array}$ & & $\begin{array}{l}-6-3 \\
-6-6 \quad P \\
(44-49)\end{array}$ & $\begin{array}{l}-7-6 \\
-8-2 \quad M \\
(73-75)\end{array}$ & 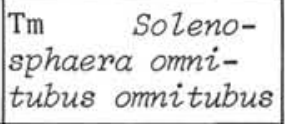 \\
\hline $\begin{array}{l}\text { Tm } \\
\text { Phormosticho- } \\
\text { artus corona }\end{array}$ & & $\begin{array}{c}\text { Below } \\
\text { A-7-5 } \\
\text { (below 232) }\end{array}$ & $\begin{array}{c}\text { Below } \\
-5-6 \\
\text { (below 76) }\end{array}$ & $\begin{array}{l}-9-1 \\
-10-1 \quad M \\
(74-84)\end{array}$ & & & $\begin{array}{l}-7-3 \\
-7-6 \quad M \\
(54-58)\end{array}$ & $\begin{array}{l}-7-6 \\
-8-4 \quad M \\
(73-78)\end{array}$ & $\begin{array}{l}\text { Tm } \\
\text { Spongaster } \\
\text { berminghami }\end{array}$ \\
\hline
\end{tabular}


TABLE 12 - Continued

\begin{tabular}{|c|c|c|c|c|c|c|c|c|c|c|}
\hline EVENTS & $\begin{array}{r}\text { SITE } \\
232\end{array}$ & $\begin{array}{r}\text { SITE } \\
233\end{array}$ & $\begin{array}{r}\text { SITE } \\
235\end{array}$ & $\begin{array}{r}\text { SITE } \\
237\end{array}$ & $\begin{array}{r}\text { SITE } \\
238\end{array}$ & $\begin{array}{c}\text { Correlation } \\
\text { lines }\end{array}$ & $\begin{array}{l}\text { SITE } \\
73\end{array}$ & $\begin{array}{r}\text { SITE } \\
77 \mathrm{~B}\end{array}$ & EVENTS & 岃 \\
\hline $\begin{array}{l}\text { Ommatartus } \\
\text { penultimus } \\
\text { o. avitus }\end{array} \rightarrow$ & & $\begin{array}{c}\text { Below } \\
\text { A-7-5 } \\
\text { (below 232) }\end{array}$ & $\begin{array}{c}\text { Below } \\
-5-6 \\
\text { (below 76) }\end{array}$ & $\begin{array}{c}-9-1 \\
-11-1 \\
(74-93)\end{array}$ & $\begin{array}{c}-13-5 \\
-14-1 \\
(117-121)\end{array}$ & & $\begin{array}{l}-7-3 \\
-7-6 \quad M \\
(54-58)\end{array}$ & $\begin{array}{c}-8-6 \\
-9-2 \\
(82-85)\end{array} \quad G$ & $\begin{array}{l}\text { Tm } \\
\text { Phormosticho- } \\
\text { artus corona }\end{array}$ & \\
\hline $\begin{array}{l}\text { Tm Soleno- } \\
\text { sphaera omni- } \\
\text { tubus omnitubus }\end{array}$ & & $\begin{array}{c}\text { Below } \\
\text { A-7-5 } \\
\text { (below 232) }\end{array}$ & $\begin{array}{c}\text { Below } \\
-5-6 \\
\text { (below 76) }\end{array}$ & $\begin{array}{c}-9-1 \\
-10-1 \\
(74-84)\end{array}$ & $\begin{array}{c}-14-1 \\
-15-1 \\
(121-130)\end{array}$ & & $\begin{array}{c}\text { Below } \\
-8-4 \\
\text { (below 63) }\end{array}$ & $\begin{array}{c}-8-6 \\
-9-2 \\
(82-85)\end{array}$ & $\begin{array}{l}\text { Spongaster } \\
\text { berminghami } \rightarrow \\
\text { S. pentas }\end{array}$ & : \\
\hline $\begin{array}{l}\text { Tm } \\
\text { Acrobotrys } \\
\text { tritubus }\end{array}$ & & $\begin{array}{c}\text { Below } \\
\text { A-7-5 } \\
\text { (below 232) }\end{array}$ & $\begin{array}{c}\text { Below } \\
-5-6 \\
\text { (below 76) }\end{array}$ & $\begin{array}{c}-10-1 \\
-11-1 \\
(84-93)\end{array}$ & $\begin{array}{c}-14-1 \\
-15-1 \\
(121-130)\end{array} P$ & & & $\begin{array}{c}-9-2 \\
-9-4 \\
(85-88)\end{array}$ & $\begin{array}{l}\text { Tm } \\
\text { Dictyocoryne } \\
\text { ontongensis }\end{array}$ & 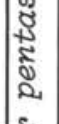 \\
\hline \multirow[t]{3}{*}{$\begin{array}{l}\text { Bm } \\
\text { Spongaster } \\
\text { pentas }\end{array}$} & $\begin{array}{l}A-19-1 \\
A-20-1 \\
(331-340)\end{array}$ & & & $\begin{array}{c}-9-1 \\
-10-1 \\
(74-84)\end{array}$ & $\begin{array}{c}-17-1 \\
-17-6 \\
(149-158)\end{array}$ & & $\begin{array}{l}-8-1 \\
-8-2 \\
(59-60)\end{array}$ & $\begin{array}{c}-9-4 \\
-9-6 \\
(88-91)\end{array}$ & $\begin{array}{l}\text { Tm } \\
\text { Acrobotrys } \\
\text { tritubus }\end{array}$ & $\begin{array}{l}0 \\
1 \\
\infty \\
8 \\
3 \\
0 \\
0 \\
0\end{array}$ \\
\hline & & & & & & & $\begin{array}{c}\text { Below } \\
-8-4 \\
\text { (below 63) }\end{array}$ & $\begin{array}{c}-9-4 \\
-9-6 \\
(88-91)\end{array}$ & $\begin{array}{l}\text { Bm } \\
\text { Spongaster } \\
\text { pentas }\end{array}$ & क \\
\hline & & & & & & & & $\begin{array}{c}-9-4 \\
-9-6 \\
(88-91)\end{array}$ & $\begin{array}{l}\text { Bm } \\
\text { Dictyocoryne } \\
\text { ontongensis }\end{array}$ & \\
\hline
\end{tabular}

Note: In the columns with the names of taxa, T indicates the top of the range of a taxon, B its bottom and an arrow an evolutionary transition. The abbreviations " $\mathrm{e}$ " and " $\mathrm{m}$ " indicate

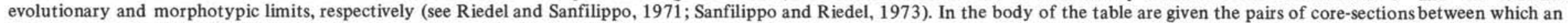

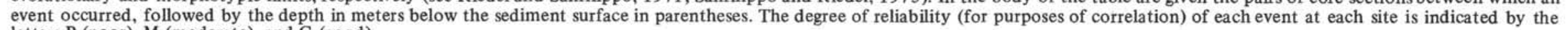
letters P (poor), M (moderate), and G (good).

This tabulation does not include the upper limits of species that range to the Recent, nor the lower limits of species ranging further back in time than latest Miocene (most of which are reported in our earlier papers in these Initial Reports). Omitted events of these types are the upper limits of Lamprocyrtis haysi, Pterocorys hertwigii, Nephrospyris renilla, Spongaster tetras, and

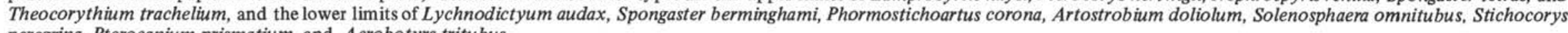
peregrina, Pterocanium prismatium, and Acrobotyrs tritubus. 


\begin{tabular}{|c|c|c|c|c|c|c|c|c|c|c|c|c|c|c|c|c|c|c|c|}
\hline 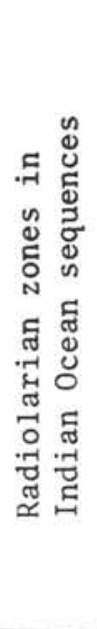 & $\begin{array}{l}\text { Approx. } \\
\text { depth } \\
\text { below } \\
\text { sediment } \\
\text { surface } \\
\text { at } \\
\text { Site } 238 \\
\quad \text { (m) }\end{array}$ & 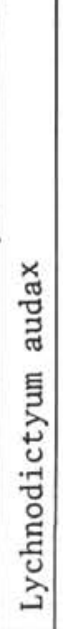 & 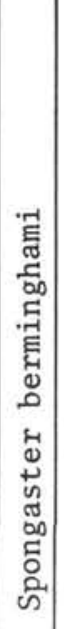 & 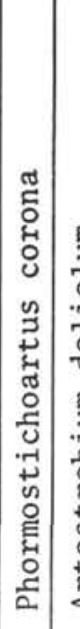 & 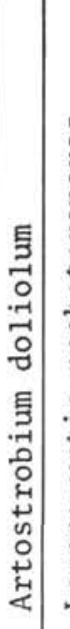 & 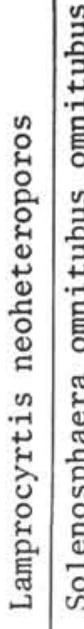 & 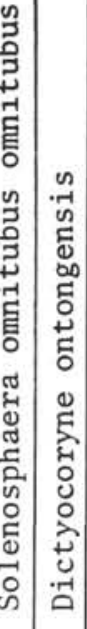 & 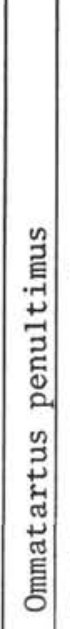 & 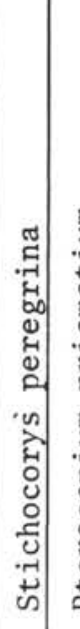 & 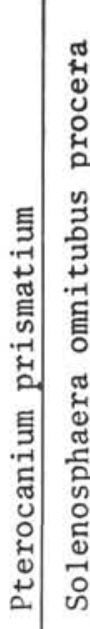 & 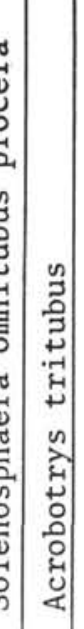 & 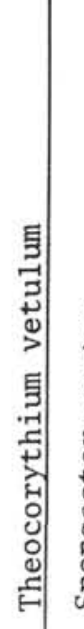 & 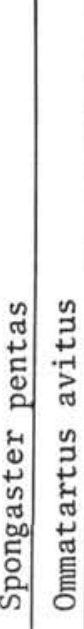 & 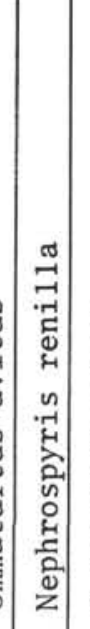 & 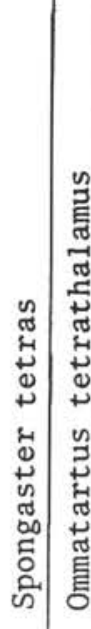 & 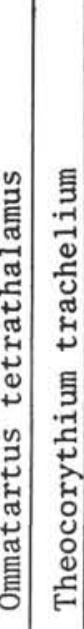 & 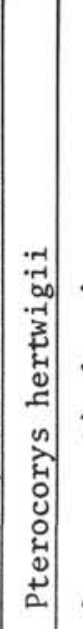 & 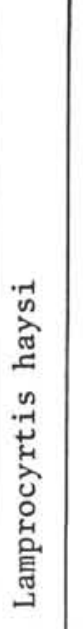 & $\begin{array}{l}\text { Approx. } \\
\text { depth } \\
\text { below } \\
\text { sediment } \\
\text { surface } \\
\text { at } \\
\text { Site } 77 \mathrm{~B} \\
\quad \text { (m) }\end{array}$ \\
\hline 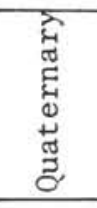 & $\begin{array}{l}10 \\
20\end{array}$ & & & & & 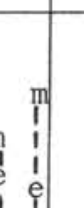 & & & & m & & & & $\left|\begin{array}{l}1 \\
1 \\
1 \\
1 \\
1 \\
1\end{array}\right|$ & \begin{tabular}{l|l|l} 
& 1 \\
1 & 1 \\
1 & 1 \\
1 & 1 \\
1 & 1 \\
1 & 1
\end{tabular} & \begin{tabular}{l|l} 
& 1 \\
1 \\
1 \\
1 \\
1 \\
1 \\
1 \\
1 \\
1
\end{tabular} & $\mid \begin{array}{l}1 \\
1 \\
1 \\
1 \\
1 \\
1 \\
1\end{array}$ & $\begin{array}{l}1 \\
1 \\
1 \\
1 \\
1 \\
1 \\
1\end{array}$ & 10 \\
\hline 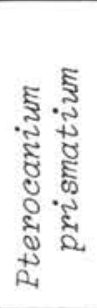 & $\begin{array}{l}30 \\
40 \\
50 \\
60\end{array}$ & & & & & $\mid \begin{array}{l}1 \\
1 \\
1 \\
v\end{array}$ & & & | & $\begin{array}{c}m \\
1 \\
1 \\
1 \\
1 \\
1 \\
1\end{array}$ & & $\begin{array}{ll}\mathrm{m} \\
\mathrm{m} & 1 \\
\mathrm{n} & \mathrm{e} \\
1 \\
1 \\
1 \\
\mathrm{e} \\
1 \\
1 \\
1 \\
1 \\
1\end{array} \mid$ & & \begin{tabular}{l|}
$i$ \\
1 \\
1 \\
1 \\
1 \\
1 \\
1 \\
1
\end{tabular} & \begin{tabular}{l|l|l} 
& 1 \\
1 & 1 \\
1 & 1 \\
1 & 1 \\
1 & 1 \\
1 & 1 \\
1 & 1 \\
1 & 1
\end{tabular} & 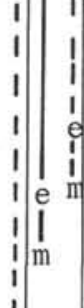 & m & $\begin{array}{l}1 \\
1 \\
1 \\
1 \\
1 \\
m\end{array}$ & 40 \\
\hline 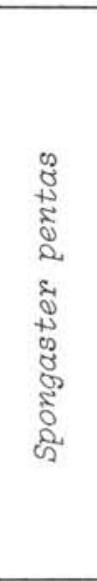 & $\begin{array}{r}70 \\
80 \\
90 \\
100 \\
110 \\
120 \\
130 \\
140\end{array}$ & 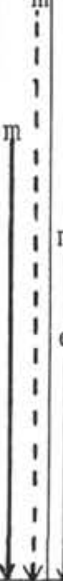 & $\mid \begin{array}{l}m \\
1 \\
1 \\
e \\
1 \\
1 \\
4\end{array}$ & 思 & $\begin{array}{l}m \\
\vdots \\
1 \\
1 \\
1 \\
1 \\
1 \\
1 \\
1 \\
1 \\
1 \\
1 \\
4\end{array}$ & & 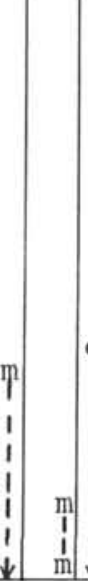 & $\left.\begin{array}{rl} & 1 \\
1 \\
0 & 1 \\
1 \\
1 \\
1 \\
1 \\
1 \\
1 \\
1 \\
1 \\
1\end{array}\right]$ & $\begin{array}{l}1 \\
1 \\
1 \\
1 \\
1 \\
1 \\
1 \\
1 \\
1 \\
1 \\
1 \\
1 \\
1 \\
1 \\
1\end{array}$ & $\begin{array}{cc}1 & \\
1 & \\
1 & \\
1 & \\
1 & m \\
1 & 1 \\
1 & 1 \\
1 & 1 \\
1 & m \\
1 & m \\
1 & 1 \\
1 & 1 \\
1 & \vdots \\
1 & \vdots \\
1 & 1 \\
1 & 1 \\
1 & 1 \\
1\end{array}$ & $\int_{0}$ & & 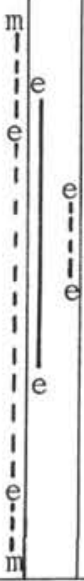 & 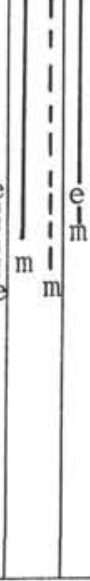 & 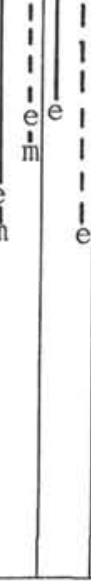 & $\begin{array}{l}1 \\
1 \\
1 \\
1 \\
1 \\
1 \\
1 \\
\mathrm{e}\end{array}$ & & & 70 \\
\hline
\end{tabular}

Figure 2. Range chart for late Neogene radiolarians in the tropical Indian and Pacific oceans. Lines indicating ranges are derived from the list of events (Table 12)-solid lines for the Indian Ocean and broken lines for the Pacific. The relation between the range lines and the depth scales (Hole 238 for the Indian Ocean and Hole 77B for the Pacific) is a compromise, taking account of evidence also from holes other than these two. The abbreviations $m$ and $e$ are used to indicate "morphotypic" and "evolutionary" limits as in our earlier papers (Riedel and Sanfilippo, 1971; Sanfilippo and Riedel, 1973), but we here conservatively record extinctions as morphotypic upper limits. 
thoracic wall, as a short spine or tube. The first post-thoracic segment is subcylindrical, approximately as wide as the thorax, and subsequent segments are inflated-annular, each with about three to six transverse rows of pores. The pores of the proximal post-thoracic segments are subcircular to elliptical, and those of the distal segments tend to be rectangular, transversely elongate. No differentiated peristome.

Measurements (based on 12 specimens from 232A-2-1, 73-5-1, and 77B-3-1). Maximum length (including horn) $170-205 \mu$; maximum breadth $75-90 \mu$.

This species is evidently closely related to the Artostrobium auritum group and to the ancestor of Spirocyrtis scalaris (Riedel and Sanfilippo, 1971, pl. 1G, figs. 19-24; pl. $2 \mathrm{H}$, figs. 15-18), from both of which it differs in being generally more robust, and in having a thickened, often thorny and irregularly pored cephalothoracic wall. The strict morphological limits of the present species definition will probably have to be modified when its relationships to closely similar forms (Pl. 4, figs. 10,11) are clarified. The specific name is an allusion to the thick-walled, rough-surfaced cephalothoracic region with prominent, irregular horn and tube.

We do not use the generic name Botryostrobus Haeckel (as do Petrushevskaya and Kozlova, 1972) because the details of the structure of the cephalis of the type species of that genus are still uncertain.

This chapter: Tab. 1-3, 6, 9-11; Sect. 3 .

Bekoma bidarfensis Riedel and Sanfilippo-Foreman, 1973, p. 432 , pl. 3 , figs. 20,21 ; pl. 10 , fig. 6 .

This chapter: Tab. 4,8 .

Bekoma campechensis Foreman, 1973, p. 342, pl. 3, fig. 24; pl. 10, figs. 1, 2; text-fig. 4 .

This chapter: Tab. 4.

Bekoma divaricata Foreman, 1973, p. 433, pl, 3, fig. 23; pl. 10 , figs. 3,4 .

This chapter: Tab. 8 .

Bekomiforma mynx Sanfilippo and Riedel, new genus and new species. Plate 2, Figures 3-5.

Cephalis with two strong horns, thorax with three strong feet, and abdomen scarcely developed. Cephalis inflated-hemispherical, with the bars of the internal spicule situated above the collar flexure; the apical bar extends beyond the shell surface as a strong spine, the vertical bar extends as a short thorn, and between them is a strong spine not related to any internal bar. Collar stricture is marked by a gentle change in contour. Thorax approximately campanulate, but this shape modified near the distal margin by the tubular extensions which pass into the three divergent, proximally latticed feet. Thoracic wall incurved terminally. Most specimens have a trace of abdominal lattice projecting from the thoracic margin, well inside the feet. Cephalis appears minutely pitted rather than porous. Pores of the thorax, abdomen, and proximal parts of the feet regular, circular, closely spaced.
Measurements (based on 10 specimens from 233-18-5, 233A-4-4, 233A-6-2, 238-4-6, and 238-11-1). Total length (including horns and feet) $125-210 \mu$. Length of thorax $60-95 \mu$.

This form, constituting a monotypic genus, is distinguished by the indistinct lower boundary to the cephalis, median bar at a higher level than the collar flexure, and one of the strong cephalic spines not associated with any internal bar. This cephalic structure is very similar to that of the genus Bekoma; the principal justification for describing the late Neogene form as a new genus is its long separation in time from that early Paleogene genus. Bekomiforma also differs in having three-bladed feet without lateral projections, and a generally less robust structure. The specific name is an arbitrary combination of letters.

Co-occurring with Bekomiforma mynx are at least two other forms with rather similar apical horns and general structure. One (Plate 2, Figure 7) has a more triangular thorax with larger, less regular pores, and the other (Plate 2, Figure 6) has a similar thoracic structure but a more pronounced collar stricture with the collar pores at the level of that stricture, as is characteristic of most theoperids. We have not yet determined the relations between these forms and B. mynx, and therefore define the latter very strictly though recognizing that the definitions of both the species and the genus may later have to be modified or broadened. This chapter: Tab. 1-3, 6, 9-11; Sect. 3 .

Botryocyrtis spp. Riedel and Sanfilippo, 1971, p. 1602, pl. $1 \mathrm{~J}$, figs. 1-11; pl. 2J, figs. 10-12; pl. 3F, fig. 7.

This chapter: Tab. 1-3, 6, 9-11.

Buccinosphaera invaginata Haeckel-Nigrini, 1971, p. 445, pl. 34.1, fig. 2 .

This chapter: Sect. 3 .

Buryella clinata Foreman, 1973, p. 433, pl. 8, figs. 1-3; pl. 9 , fig. 19.

This chapter: Tab. 8 .

Buryella pentadica Foreman, 1973, p. 433, pl. 8, fig. 8; pl. 9 , figs. $15,16$.

This chapter: Tab. 4,8 .

Buryella tetradica Foreman, 1973, p. 433, pl. 8, figs. 4, 5; pl. 9, figs. 13, 14.

This chapter: Tab. 5,8 .

Calocyclas hispida (Ehrenberg)-Foreman, 1973, p. 434, pl. 1 , figs. $12-15$; pl. 9 , fig. 18 .

This chapter: Tab. $5,7,8$.

Calocyclas turris Ehrenberg-Riedel and Sanfilippo, 1970, p. 529, pl. 13, figs. 3, 4 (as Cycladophora turris).

This chapter: Tab. 5 .

Calocycletta caepa Moore, 1972, p. 150, pl. 2, figs. 4-7. This chapter: Tab. 4, 9-11; Pl. 3, fig. 8. 
Calocycletta costata (Riedel)-Riedel and Sanfilippo, 1970, p. 535 , pl. 14, fig. 12 .

This chapter: Tab. 9.

Calocyletta virginis Haeckel-Moore, 1972, p. 147, pl. 1, fig. 7 .

This chapter: Tab. 9, 10.

Calocycloma castum (Haeckel)-Foreman, 1973, p. 434, pl. 1 , figs. $7,9,10$.

This chapter: Tab. 8 .

Cannartus laticonus Riedel, 1959, p. 291, pl. 1, fig. 5. This chapter: Tab. 9.

Cannartus mammifer (Haeckel)-Riedel, 1959, p. 291, pl. 1, fig. 4.

This chapter: Tab. 9.

Cannartus (?) petterssoni Riedel and Sanfilippo, 1970, p. 520 , pl. 14, fig. 3 .

This chapter: Tab. 9.

Cannartus sp. cf. C. prismaticus (Haeckel). Plate 1, figure 7.

Under this name we record a form very closely resembling $C$. prismaticus, but with probably a different phyletic origin. The cortical ellipsoid is almost spherical, the external medullary shell is lenticular, and the spongy columns are commonly broken off (never observed to be long). It appears to have been preceded (and during the early part of its range accompanied) by a form with a shallow equatorial constriction and rather acute protuberances on the cortical shell (Plate 1, Figures 8, 9).

Its stratigraphic range is evidently not continuous with that of $C$. prismaticus. Rather similar forms have been described from the Italian Neogene (e.g., Cannartiscus canavarii Vinassa), but these are pronouncedly ellipsoidal and have longer polar columns, and are probably identical with $C$. prismaticus.

This chapter: Tab. 6, 9 (with footnotes); Plate 1, Figure 7.

Carpocanarium spp. Riedel and Sanfilippo, 1971, p. 1599, pl. 11, figs. $17-25$; pl. 2 J, figs 8,9 .

This chapter: Tab. 1-4, 6, 9-11.

Carpocanopsis bramlettei Riedel and Sanfilippo, 1971, p. 1597, pl. 2G, figs. 8-14; pl. 8, fig. 7.

This chapter: Tab. 9.

Carpocanopsis cingulata Riedel and Sanfilippo, 1971, p. 1597, pl. 2G, figs. $17-21$; pl. 8 , fig. 8 .

This chapter: Tab. 9 (with footnote); Plate 3, Figure 7.

Carpocanopsis favosa (Haeckel)-Riedel and Sanfilippo, 1971, p. 1597, pl. 2G, figs. 15, 16; pl. 8, figs. 9-11.

This chapter: Tab. 9, 10.
Centrobotrys petrushevskayae Sanfilippo and Riedel, 1973, p. 532 , pl. 36 , figs. $12,13$.

This chapter: Tab. 9.

Centrobotrys thermophila Petrushevskaya-Nigrini, 1967, p. 49 , text-fig. 26 , pl. 5 , fig. 7 .

This chapter: Tab. 1-3, 6, 9-11.

Ceratospyris articulata Ehrenberg-Sanfilippo and Riedel, 1973 , p. 526 , pl. 15 , figs. $1-3$; pl. 3 I, figs. 8,9 .

This chapter: Tab. $5,7,8$.

Clathrocycloma capitaneum Foreman, 1973, p. 434, pl. 2, fig. $15 ;$ pl. 11, fig. 11 .

This chapter: Tab. 8 .

Cyclampterium (?) neatum Sanfilippo and Riedel, 1970, p. 457 , pl. 2 , figs. 17,18 .

This chapter: Tab. 1-4, 6, 9 (with footnote), 10, 11 .

Cyrtocapsella cornuta Haeckel-Sanfilippo and Riedel, 1970 , p. 453, pl. 1, figs. 19, 20.

This chapter: Tab. $9,10$.

Cyrtocapsella tetrapera Haeckel-Sanfilippo and Riedel, 1970 , p. 453, pl. 1, figs. 16-18.

This chapter: Tab. 4, 9 .

Dendrospyris acuta Goll-Sanfilippo and Riedel, 1973, p. 526, pl. 15, fig. 5; pl. 31, fig. 11.

This chapter: Tab. 8.

Dendrospyris bursa Sanfilippo and Riedel, in Sanfilippo et al., in press.

This chapter: Tab. 9.

Dendrospyris fragoides Sanfilippo and Riedel, 1973, p. 526, pl. 15, figs. 8-13; pl. 31, figs. 13, 14 .

This chapter: Tab. 8 .

Dictyocoryne ontongensis Riedel and Sanfilippo, 1971, p. 1588 , pl. 1E, figs. 1,2 ; pl. 4, figs. 9-11.

This chapter: Tab. 9-12; Text-fig. 2 .

Dictyophimus craticula Ehrenberg-Sanfilippo and Riedel, 1973 , p. 529 , pl. 19 , fig. 1 ; pl. 33, fig. 11.

This chapter: Tab. 7.

Dictyospyris gigas Ehrenberg-Sanfilippo and Riedel, 1973, p. 527 , pl. 16 , figs. 9,10 ; pl. 32 , figs. 10,11 .

This chapter: Tab. 8.

Dorcadospyris alata (Riedel)-Riedel and Sanfilippo, 1970, p. 523 , pl. 14 , fig. 5 .

This chapter: Tab. 9 (with footnote).

Dorcadospyris dentata Haeckel-Riedel, 1957, p. 79, pl. 1, fig. 4 .

This chapter: Tab. 9 (with footnote), 10 . 
Dorcadospyris spinosa Moore, 1971, p. 739, pl. 7; figs. 1, 2. This chapter: Tab. 5 .

Eusyringium fistuligerum (Ehrenberg)-Foreman, 1973, p. 435 , pl. 11, fig. 6 .

This chapter: Tab. 7.

Eusyringium lagena (Ehrenberg)-Foreman, 1973, p. 435, pl. 11, figs. 4,5 .

This chapter: Tab. 7,8 .

Giraffospyris lata Goll-Sanfilippo and Riedel, 1973, p. 529 , pl. 18, figs. 3-7; pl. 33, fig. 4 .

This chapter: Tab. 5,8 .

Heliostylus spp. Sanfilippo and Riedel, 1973, p. 522, pl. 8, figs. $1-7$; pl. 26 , figs. $10-12$; pl. 27 , fig. 1 .

This chapter: Tab. 8.

Lamprocyrtis haysi Kling, 1973, p. 639, pl. 5, figs. 15, 16; pl. 15 , figs. 1-3.

This chapter: Tab. 1-4, 6, 9-12; Text-fig. 2; Sect. 3; Plate 3, Figures 9, 10.

Lamprocyrtis neoheteroporos Kling, 1973, p. 639, pl. 5, figs. 17,$18 ;$ pl. 15 , figs. 4,5 .

This chapter: Tab. 1-4, 6, 9-12; Text-fig. 2; Sect. 3; Pl. 3 , fig. 11 .

Lamptonium fabaeforme fabaeforme (Krasheninnikov) (?)-Foreman, 1973, p. 436, pl. 6, figs. 6-9.

This chapter: Tab. 7,8 .

Lamptonium fabaeforme (?) chaunothorax Riedel and Sanfilippo, 1970 , p. 524, pl. 5, figs. 8,9 .

This chapter: Tab. 7, 8 .

Lamptonium fabaeforme (?) constrictum Riedel and Sanfilippo, 1970, p. 523, pl. 5, fig. 7.

This chapter: Tab. 7.

Lamptonium pennatum Foreman, 1973, p. 436, pl. 6, figs. 3-5; pl. 11, fig. 13.

This chapter: Tab. 8 .

Lamptonium sanfilippoae Foreman, 1973, p. 436, pl. 6, figs. 15,16 ; pl. 11, figs. 16, 17 .

This chapter: Tab. 7.

Liriospyris elevata Goll, 1968, p. 1426, pl. 175, figs. 4, 5, 8, 9; text-fig. 9.

This chapter: Tab. 9.

Liriospyris stauropora (Haeckel)-Goll, 1968, p. 1431, pl. 175, figs. $1-3,7$; text-fig. 9 .

This chapter: Tab. 9, 10.

Lithapium anoectum Riedel and Sanfilippo, 1970, p. 520, pl. 4 , figs. 4,5 .

This chapter: Tab. 7.
Lithapium mitra (Ehrenberg)-Riedel and Sanfilippo, 1970, p. 520 , pl. 4 , figs. 6,7 .

This chapter: Tab. 7.

Lithapium plegmacantha Riedel and Sanfilippo-Sanfilippo and Riedel, 1973, p. 516, pl. 3, figs. 1, 2; pl. 24, figs. 8, 9 .

This chapter: Tab. 5,7 .

Lithochytris archaea Riedel and Sanfilippo-Foreman, 1973, p. 436, pl. 2, figs. 4, 5 .

This chapter: Tab. 7, 8 .

Lithochytris vespertilio Ehrenberg-Riedel and Sanfilippo, 1970 , p. 528 , pl. 9 , figs. 8,9 .

This chapter: Tab. 7.

Lithocyclia angusta (Riedel)-Riedel and Sanfilippo, 1970, p. 522, pl. 13, figs. $1,2$.

This chapter: Tab. 5 .

Lithocyclia aristotelis (Ehrenberg) group-Riedel and Sanfilippo, 1970, p. 522.

This chapter: Tab. 7 (with footnote).

Lithocyclia ocellus Ehrenberg group-Riedel and Sanfilippo, 1970, p. 522, pl. 5, figs. 1, 2.

This chapter: Tab. $4,7$.

Lithopera bacca Ehrenberg-Nigrini, 1967, p. 54, pl. 6, fig. 2.

This chapter: Tab. 1-3, 6, 9-11.

Lithopera neotera Sanfilippo and Riedel, 1970, p. 454, pl. 1 , figs. 24-26, 28.

This chapter: Tab. 9.

Lophocyrtis biaurita (Ehrenberg)-Cita et al., 1970, p. 404, pl. 2, figs. I, J, K.

This chapter: Tab. 7, 8.

Lychnocanoma amphitrite Foreman, 1973, p. 437, pl. 11, fig. 10.

This chapter: Tab. 5 .

Lychnocanoma auxilla Foreman, 1973, p. 437, pl. 2, fig. 6; pl. 11, figs. 1, 2 .

This chapter: Tab. 4,8 .

Lychnocanoma bellum (Clark and Campbell)-Foreman, 1973, p. 437, pl. 1, fig. 17, pl. 11, fig. 9.

This chapter: Tab. 7.

Lychnocanoma elongata (Vinassa)-Sanfilippo and Riedel in Sanfilippo et al., in press.

This chapter: Tab. 4.

Lychnodictyum audax Riedel, 1953, p. 810, pl. 85, fig. 9 . Although this species is evidently closely related to 
stratigraphically useful forms such as Pterocanium prismatium, it is not now transferred to that genus because of uncertainty regarding the nature of the unfigured type species of Pterocanium (Lithocampe aculeata Ehrenberg, according to Foreman and Riedel, 1972).

This chapter: Tab. 1-4, 6, 9-12; Text-fig. 2; Sect. 3; Plate 2, Figure 1.

Nephrospyris renilla Haeckel-Goll, 1972, p. 967, pl. 72, figs. 1-3.

This chapter: Tab. 1-4, 6, 9-12; Text-fig. 2; Sect. 3; Plate 2, Figure 1.

Ommatartus antepenultimus Riedel and Sanfilippo, 1970, p. 521 , pl. 14, fig. 4 .

This chapter: Tab. 1, 3, 4, 9-11.

Ommatartus avitus (Riedel)-Riedel and Sanfilippo, 1971, p. 1588 , pl. 4 , fig. 6 .

This chapter: Tab. 1-4, 6, 9-12; Text-fig. 2; Sect. 3; Plate 1, Figure 10.

Ommatartus hughesi (Campbell and Clark)-Riedel and Sanfilippo, 1970, p. 521.

This chapter: Tab. 1 (with footnote), 4, 9, 11 .

Ommatartus penultimus (Riedel)-Riedel and Funnell, 1964, p. 311.

This chapter: Tab. 1, 2, 4, 6, 9-12; Text-fig. 2 .

Ommatartus tetrathalamus (Haeckel)-Riedel and Sanfilippo, 1971, p. 1588, pl. 1C, figs. 5-7.

This Chapter: Tab. 1-4, 6, 9-12; Text-fig. 2; Sect. 3.

Periphaena tripyramis triangula (Sutton)-Sanfilippo and Riedel, 1973, p. 523, pl. 9, figs. 10, 11.

This chapter: Tab. 7.

Phormocyrtis cubensis (Riedel and Sanfilippo)-Foreman, 1973 , p. 438, pl. 7, figs. 11, 12, 14.

This chapter: Tab. 8 (with footnote).

Phormocyrtis striata striata Brandt-Foreman, 1973, p. 438, pl. 7, figs. 5, 6, 9 .

This chapter: Tab. $5,7,8$.

Phormocyrtis striata exquisita (Kozlova)-Foreman, 1973, p. 438 , pl. 7 , figs. $1-4,7,8$; pl. 12 , fig. 5 .

This chapter: Tab. 5,8 .

Phormocyrtis turgida (Krasheninnikov)-Foreman 1973, p. 438 , pl. 7 , fig. 10 ; pl. 12 , fig. 6 .

This chapter: Tab. 4,8 .

Phormostichoartus corona Haeckel-Riedel and Sanfilippo, 1971 , p. 1600 , pl. 1I, figs. 13-15; pl. 2J, tigs. 1-5.

This chapter: Tab. 1-3, 6, 9-12; Text-fig. 2 .

Podocyrtis ampla ampla Ehrenberg-Riedel and Sanfilippo, 1970 , p. 533, pl. 12 , figs. 7,8 .

This chapter: Tab. 7.
Podocyrtis diamesa Riedel and Sanfilippo-Sanfilippo and Riedel, 1973, p. 531, pl. 20, figs. 9, 10; pl. 35, figs. 10, 11.

This chapter: Tab. 7 .

Podocyrtis dorus Sanfilippo and Riedel, 1973, p. 531, pl. 35, figs. 12-14.

This chapter: Tab. 7.

Podocyrtis ampla fasciolata Nigrini, in press. This chapter: Tab. 5,7 .

Podocyrtis goetheana (Haeckel)-Riedel and Sanfilippo, 1970 , p. 535.

This chapter: Tab. 5 .

Podocyrtis helenae Nigrini, in press.

This chapter: Tab. 7.

Podocyrtis mitra Ehrenberg-Riedel and Sanfilippo, 1970, p. 534 , pl. 11 , figs. 5,6 .

This chapter: Tab. 4, 5, 7 .

Podocyrtis papalis Ehrenberg-Riedel and Sanfilippo, 1970, p. 533 , pl. 11 , fig. 1 .

This chapter: Tab. 7, 9 (with footnote).

Podocyrtis sinuosa Ehrenberg-Riedel and Sanfilippo, 1970 p. $534, \mathrm{pl}, 11$, figs. 3,4 .

This chapter: Tab. 4, 5, 7, 8 .

Podocyrtis trachodes Riedel and Sanfilippo, 1970, p. 535, pl. 11, fig. 7 ; pl. 12, fig. 1 .

This chapter: Tab. 4, 7.

Pterocanium prismatium Riedel-Riedel and Sanfilippo, 1970 , p. 529.

This chapter: Tab. 1-4, 6 (with footnote), 9-12, Text-fig. 2; Sect. 3 .

Pterocodon tenellus Foreman, 1973, p. 439, pl. 5, fig. 7; pl. 12 , fig. 4 .

This chapter: Tab. 5,8 .

Pterocorys hertwigii (Haeckel)-Petrushevskaya, 1972, fig. 1 (10).

This chapter: Tab. 1-4, 6, 9-12; Text-fig. 2; Sect. 3; Plate 3, Figures 12-14.

Rhopalocanium ornatum Ehrenberg-Foreman, 1973, p. 439 , pl. 2, figs. $8-10$; pl. 12, fig. 3.

This chapter: Tab. 7 .

Sethochytris babylonis (Clark and Campbell) group-Riedel and Sanfilippo, 1970, p. 528, pl. 9, figs. 1-3.

This chapter: Tab. 7, 8 .

Sethochytris triconiscus Haeckel-Riedel and Sanfilippo, 1970 , p. 528 , pl. 9 , fig. 6 .

This chapter: Tab. 5, 7 . 
Siphocampe corbula (Harting)-Riedel and Sanfilippo, 1971 , p. 1601 , pl. $1 \mathrm{H}$, figs. $18-25$.

This chapter: Tab. 1-4, 6, 9-11.

Solenosphaera omnitubus omnitubus Riedel and Sanfilippo, 1971 , p. 1586, pl. 1A, fig. 24; figs. 1, 2.

We would now exclude from this species the form illustrated in fig. 23 of pl. 1A of the above-cited paper, since the bases of the tubular prolongations constitute too small a proportion of the spherical shell. As the nominate subspecies, we now record specimens in which the tubular prolongations are as short as, or shorter than, those in the forms illustrated in 1971 excluding fig. 23 .

This chapter: Tab. 1-4, 6 (with footnote), 9, 10, 11 (with footnote), 12; Text-fig. 2; Plate 1, Figure 1.

Solenosphaera omnitubus procera Sanfilippo and Riedel, new subspecies. Plate 1, Figures 2-5.

This subspecies comprises specimens with three to six tubular prolongations, longer than those of the nomiate subspecies, and no spherical central shall.

Maximum diameter, to ends of tubes (based on 10 specimens from 238-15-6, 238-16-1, 77B-8-2, and 77B-8-4), 55-105 $\mu$.

The subspecific name is derived from the Latin adjective meaning "long."

This chapter: Tab. 1-3, 6, 9-12; Text-fig. 2; Plate 1, Figures 2-5.

Spirocyrtis scalaris Haeckel-Riedel and Sanfilippo, 1971, p. 1601, pl. $1 \mathrm{G}$, figs. $25-27$.

This chapter: Tab. 1-4, 6, 9-11.

Spongaster berminghami (Campbell and Clark)-Sanfilippo and Riedel, 1973, p. 524 [8(6)C].

This chapter: Tab. 1, 2, 4, 6, 9-12; Text-fig. 2 .

Spongaster pentas Riedel and Sanfilippo, 1970, p. 523, pl. 15 , fig. 3 .

This chapter: Tab. 1-3, 6, 9-12; Text-fig. 2

Spongaster tetras Ehrenberg-Riedel and Sanfilippo, 1971, p. 1589 , pl. 1D, figs. $2-4$.

This chapter: Tab. 1-4, 6, 9-12; Text-fig. 2 .

Spongatrachtus pachystylus (Ehrenberg)-Sanfilippo and Riedel, 1973, p. 519, pl. 2, figs. 4-6; pl. 25, fig. 3.

This chapter: Tab. 7.

Spongodiscus ambus Sanfilippo and Riedel, new species. Plate 1, Figures 12-14.

A delicate, subtriangular or less commonly circular disc, often with one margin indented, and with prominent, eye-like center. The major part of the rounded-triangular skeleton is of rather loose, irregular spongy meshwork, of different thickness in different parts of the disc. The darker central portion is formed of four to six closely spaced spiral to concentric whorls. In rare specimens, three differentiated rays depart from this central portion in the directions of the corners of the disc. In some, a hollow cone extends inward from one side. No differentiated margin.

Measurements (based on 25 specimens from 232A$20-1$ and 77B-7). Maximum diameter $170-485 \mu$ (usually about $360 \mu)$.

This species differs from others in the genus by its irregular structure and marked tendency to triangular outline. The species name is an arbitrary combination of letters.

There is some similarity to Schizodiscus Dogiel, but species of that genus (Dogel and Reshetnyak, 1952) have a surficial lattice-plate, no tendency to triangularity, and commonly a differentiated margin.

This chapter: Tab. 1 (with footnote), 9, 10 (with footnote), 11; Sect. 3 .

Spongodiscus cruciferus Clark and Campbell-Sanfilippo and Riedel, 1973, p. 524, pl. 11, figs. 14-17; pl. 28, figs. 10, 11.

This chapter: Tab. 8 .

Spongodiscus phrix Sanfilippo and Riedel, 1973, p. 525, pl. 12 , figs. 1,2 ; pl. 29 , fig. 2 .

This chapter: Tab. 7.

Spongodiscus quartus quartus (Borisenko)-Sanfilippo and Riedel, 1973, p. 525, pl. 12, figs. 3-5; pl. 29, figs. 5-6.

This chapter: Tab. 8.

Stichocorys delmontensis (Campbell and Clark)-Sanfilippo and Riedel, 1970, p. 451 , pl. 1 , fig. 9.

This chapter: Tab. 1, 2, 4, 6, 9-11.

Stichocorys peregrina (Riedel)-Sanfilippo and Riedel, 1970 , p. 451, pl. 1, fig. 10.

This chapter: Tab. 1-4, 6, 9-12; Text-fig. 2 .

Stichocorys wolffii Haeckel-Riedel, 1957, p. 92, pl. 4, figs. $6,7$.

This chapter: Tab. 1,9.

Stylotrochus alveatus Sanfilippo and Riedel, 1973, p. 525, pl. 13 , figs. 4,5 ; pl. 30 , figs. 3,4 .

This chapter: Tab. 8 .

Stylotrochus nitidus Sanfilippo and Riedel, 1973, p. 525, pl. 13, figs. 9-14; pl. 30, figs. 7-10.

This chapter: Tab. 8 .

Stylotrochus quadribrachiatus quadribrachiatus Sanfilippo and Riedel, 1973 , p. 526, pl. 14, figs. 1, 2; pl. 31. fig. 1. This chapter: Tab. 8 .

Tepka perforata Sanfilippo and Riedel, in Sanfilippo et al., in press.

This chapter: Tab. 9.

Thecosphaera larnacium Sanfilippo and Riedel, 1973, p. 521, pl. 3, figs. 4-6; pl. 25, figs. 13, 14.

This chapter: Tab. 8 . 
Theocampe amphora (Haeckel) group-Foreman, 1973, p. 431 , pl. 8 , figs. $7,9-13$; pl. 9 , figs. 8,9 .

This chapter: Tab. 7 .

Theocampe mongolfieri (Ehrenberg)-Foreman, 1973, p. 432, pl. 9, fig. 6; pl. 9, fig. 17.

This chapter: Tab. 5, 7 .

Theocampe pirum (Ehrenberg)-Riedel and Sanfilippo, 1971 , p. 1601 , pl. $3 \mathrm{E}$, figs. $10,11$.

This chapter: Tab. 7.

Theocampe urceolus (Haeckel)-Foreman, 1973, p. 432, pl. 8, figs. 14-17; pl. 9, figs. 6,7 .

This chapter: Tab. 8.

Theocorys anapographa Riedel and Sanfilippo-Foreman, 1973, p. 440, pl. 5, figs. 9, 10.

This chapter: Tab 7 (with footnote).

Theocorys phyzella Foreman, 1973, p. 440, pl. 5, fig. 8; pl. 12 , fig. 1.

This chapter: Tab. 4,8 .

Theocorys spongoconum Kling, 1971, p. 1087, pl. 5, fig. 6. This chapter: Tab. 4.

Theocorythium trachelium (Ehrenberg)-Nigrini, 1967, p. 77, pl. 9, figs. 1,2 ; pl. 8, figs. 1,2 .

This chapter: Tab. 1-4, 6, 9-12; Text-fig. 2; Sect. 3; Plate 4, Figures 3-5.

Theocorythium vetulum Nigrini, 1971, p. 447, pl. 34.1, figs. $6 a, b$.

This chapter: Tab. 1-4, 6, 9-12; Text-fig. 2; Sect. 3; Plate 4, Figures 6, 7.

Theocotyle auctor Foreman, 1973, p. 441, pl. 4, figs. 8-10; pl. 12 , fig. 13 .

This chapter: Tab. 8 .

Theocotyle cryptocephala cryptocephala (Ehrenberg)Foreman, 1973, p. 440, pl. 4, figs. 6, 7; pl. 12, fig. 18 This chapter: Tab. 7.

Theocotyle cryptocephala conica Foreman, 1973, p. 440, pl. 4, fig. 11 ; pl. 12, figs. 19, 20.

This chapter: Tab. 7.

Theocotyle cryptocephala nigriniae Riedel and SanfilippoForeman, 1973, p. 440, pl. 4, figs. 1, 3-5 (perhaps not 2); pl. 12, fig. 17 .

This chapter: Tab. 5,8 .

Theocotyle ficus (Ehrenberg)-Foreman, 1973, p. 441, pl. 4 , figs. 16-20.

This chapter: Tab. 5, 7 .

Theocotyle fimbria Foreman, 1973, p. 441, pl. 5, figs. 1, 2; pl. 12 , fig. 21 .

This chapter: Tab. 8 .
Theocyrtis tuberosa Riedel, 1959, p. 298, pl. 2, figs. 10, 11. This chapter: Tab. 5 .

Thyrsocyrtis bromia Ehrenberg-Riedel and Sanfilippo, 1971 , p. 1596 , pl. 8 , fig. 6 .

This chapter: Tab. 5 .

Thyroscyrtis hirsuta hirsuta (Krasheninnikov)-Foreman, 1973 , p. 441 , pl. 3 , figs. $3-8$; pl. 12 , fig. 15

This chapter: Tab. 5, 7,8.

Thyrsocyrtis hirsuta robusta Riedel and SanfilippoForeman, 1973, p. 442, pl. 3, fig. 17.

This chapter: Tab. 7.

Thyrsocyrtis hirsuta tensa Foreman, 1973, p. 442, pl. 3, figs. $13-16$; pl. 12, fig. 8 .

This chapter: Tab. 7 .

Thyrsocyrtis rhizodon Ehrenberg-Foreman, 1973, p. 442, pl. 3 , figs. 1,2 .

This chapter: Tab. 5,7 .

Thyrsocyrtis tetracantha (Ehrenberg)-Riedel and Sanfilippo, 1970, p. 527.

This chapter: Tab. 4, 5 .

Thyrsocyrtis triacantha (Ehrenberg)-Foreman, 1973, p. 442 , pl. 12, figs. 9-11.

This chapter: Tab. 4, 5, 7 .

Tricolocampe vitrea Krasheninnikov-Foreman, 1973, p. 438 , pl. 7, fig. 13.

This chapter: Tab. 8 (footnote); Plate 3, Figure 4.

Tristylospyris triceros (Ehrenberg)-Haeckel, 1887, p. 1033.

This chapter: Tab. 5 .

\section{ACKNOWLEDGMENTS}

Financial support for this study was provided partly by NSF Grant No. GA-31284X and partly by the University of California. The onerous tasks of typing and proofreading were competently performed by M. A. Hanger and M. A. Neely, and the substantial contribution by David C. Clegg has been mentioned in the Introduction. For providing the foraminiferal information used in constructing Figure 1, we are grateful to Edith Vincent.

\section{REFERENCES}

Cita, M. B., Nigrini, C., and Gartner, S., 1970. Biostratrigraphy. In Peterson, M. N. A. et al., Initial Reports of the Deep Sea Drilling Project, Volume II: Washington (U.S. Government Printing Office), p. 391-411.

Dogel, V. A. and Reshetnyak, V. V., 1952. Materialy po radiolyariyam severozapadnoi chasti Tikhogo okeana; Issledovaniya Dalnevostochnykh Morei SSSR, v. 3, p. 5-36.

Foreman, H. P., 1973. Radiolaria of Leg 10 with systematics and ranges for the families Amphipyndacidae, Artostrobiidae, and Theoperidae. In Worzel, 
J. L., Bryant, W., et al., Initial Reports of the Deep Sea Drilling Project, Volume X: Washington (U. S. Government Printing Office), p. 407-474.

Goll, R. M., 1972. Systematics of eight Tholospyris taxa (Trissocyclidae, Radiolaria): Micropaleontology, v. 18, no. 4 , p. 443-475.

Haeckel, E., 1887. Report on the Radiolaria collected by H.M.S. Challenger during the years 1873-76. Rept. Voyage Challenger, Zool, v. 18.

Hays, J. D., 1965. Radiolaria and late Tertiary and Quaternary history of Antarctic seas: Biol. Antarctic Seas, v. 2, Antarctic Res. Ser. 5 (Am. Geophys. Union), p. $125-184$.

1970. Stratigraphy and evolutionary trends of Radiolaria in North Pacific deep-sea sediments: Geol. Soc. Am., Mem. 126, p. 185-218.

1971. Faunal extinctions and reversals of the Earth's magnetic field: Geol. Soc. Am. Bull., v. 82, p. 2433-2447.

Kling, S. A., 1971. Radiolaria: Leg 6 of the Deep Sea Drilling Project. In Fischer, A. G., et al., Initial Reports of the Deep Sea Drilling Project, Volume VI: Washington (U.S. Government Printing Office), p. 1069-1117.

1973. Radiolaria from the eastern North Pacific, Deep Sea Drilling Project Leg 18. In Kulm, L. D., von Huene, R., et al., Initial Reports of the Deep Sea Drilling Project, Volume XVIII: Washington (U. S. Government Printing Office), p. 617-671.

Moore, T. C., Jr., 1971. Radiolaria. In Tracey, J. I. Jr. et al., Initial Reports of the Deep Sea Drilling Project, Volume VIII: Washington (U. S. Government Printing Office), p. 727-775.

1972. Mid-Tertiary evolution of the radiolarian genus Calocycletta: Micropaleontology, v. 18, p. 144-152.

Nigrini, C., 1967. Radiolaria in pelagic sediments from the Indian and Atlantic Oceans: Bull. Scripps Inst. Oceanog. Univ. Calif., v. 11, 125 p.

1971. Radiolaria zones in the Quaternary of the equatorial Pacific Ocean. In Funnell, B. M., and Riedel, W. R., (Eds.) The micropalaeontology of oceans: Cambridge (Cambridge Univ. Press), p. 443-461.

in press. Cenozoic Radiolaria from the Arabian Sea, DSDP Leg 23. In Whitmarsh, R. B., Weser, O. E., Ross, D. A., et al., Initial Reports of the Deep Sea Drilling Project, Vol XXIII: Washington (U.S. Government Printing Office).
Petrushevskaya, M. G., 1972. Biostratigrafiya glubokovodnykh chetvertichnykh osadkov po dannym radiolyarievogo analyza: Okeanologiya, v. 12, no. 1, p. 71-86.

Petrushevskaya, M. G. and Kozlova, G. E., 1972. Radiolaria: Leg 14, Deep Sea Drilling Project. In Hayes, D. E., Pimm, A. C., et al., Initial Reports of the Deep Sea Drilling Project, Volume XIV: Washington (U.S. Government Printing Office), p. 495-648.

Riedel, W. R., 1957. Radiolaria: a preliminary stratigraphy: Rep. Swed. Deep-Sea Exped., v. 6, no. 3, p 59-96. 1959. Oligocene and Lower Miocene Radiolaria in tropical Pacific sediments: Micropaleontology, v. 5, no. 3 , p. $285-302$.

Riedel, W. R, and Foreman, H. P., 1961. Type specimens of North American Paleozoic Radiolaria: J. Paleontol., v. 35 , no. 3, p. 628-632.

Riedel, W. R., and Funnell, B. M., 1964. Tertiary sediment cores and microfossils from the Pacific Ocean floor: Geol. Soc. London, Quart. J., v. 120, p. 305-368.

Riedel, W. R. and Sanfilippo, A., 1970. Radiolaria, Leg 4, Deep Sea Drilling Project. In Bader, R. G. et al., Initial Reports of the Deep Sea Drilling Project, Volume IV; Washington (U.S. Government Printing Office), p. 503-575.

1971. Cenozoic Radiolaria from the western tropical Pacific, Leg 7. In Winterer, E. L. et al., Initial Reports of the Deep Sea Drilling Project, Volume VII: Washington (U.S. Government Printing Office), p. 1529-1672.

, in press. Cenozoic Radiolaria from the Caribbean, DSDP Leg 15. In Edgar, N. T., Saunders, J. B., et al., Initial Repts. of the Deep Sea Drilling Project, Volume XV: Washington (U. S. Government Printing Office).

Sanfilippo, A., Burckle, L. H., Martini, E., and Riedel, W. R., in press. Radiolarians, diatoms, silicoflagellates and calcareous nannofossils in the Mediterranean Neogene: Micropaleontology.

Sanfilippo, A., and Riedel, W. R., 1970. Post-Eocene "closed" theoperid radiolarians: Micropaleontology, v. 16 , no. 4, p. 446-462.

1973. Cenozoic Radiolaria (exclusive of theoperids, artostrobiids and amphipyndacids) from the Gulf of Mexico, DSDP Leg 10. In Worzel, J. L., Bryant, W., et al., Initial Reports of the Deep Sea Drilling Project, Volume X: Washington (U.S. Government Printing Office), p. 475-611.

In the explanations to the figures, the sample numbers and slide designations (in the form "Ph.2," "Sl.1," "Cse.2," etc.) indicate preparations in our collection at Scripps Institution of Oceanography (or, in the case of type specimens, preparations to be deposited in the U. S. National Museum, Washington, D. C.), and designations in the form "R45/1" indicate England Finder positions of the illustrated specimens on the slides (Riedel and Foreman, 1961). 



\section{PLATE 1}

(Unless otherwise indicated, magnifications are 225X)

Figure $1 \quad$ Solenosphaera omnitubus omnitubus 237-11-1, 48-52 cm, Ph.2, F27/2.

Figures 2-5 Solenosphaera omnitubus procera Sanfilippo and Riedel, new subspecies.

2. $73-6-3,130-132 \mathrm{~cm}, \mathrm{Sl} .1, \mathrm{M} 15 / 0$.

3. (holotype), 73-6-3, 130-132 cm, Sl.1, F38/1.

4. 233-15-6, 99-102 cm, Sl.2, D27/3.

5. 77B-8-2, 6-8 cm, Ph.1, S21/3.

Figure 6 Solenosphaera sp., excluded from $S$. omnitubus procera because the central body is too well developed. 237-10-1, 97-101 cm, S1.1, T31/1.

Figure $7 \quad$ Cannartus sp. cf. C. prismaticus 238-23-6, 98-101 cm, Sl.2, N12/3.

Figures 8, $9 \quad$ Cannartus sp.

8. $238-19-6,98-101 \mathrm{~cm}$, Sl. $2, \mathrm{P} 30 / 4$.

9. 238-23-6, $98-101 \mathrm{~cm}, \mathrm{Sl} .2, \mathrm{H} 15 / 0$.

Figure $10 \quad$ Ommatartus avitus

237-8-1, 50-54 cm, S1.1, Q44/3.

Figure $11 \quad$ Coccodiscid sp. cf. Lithocyclia aristotelis group 237-25-1, 48-52 cm, Sl.1, N22/0.

Figures 12-14 Spongodiscus ambus Sanfilippo and Riedel, new species.

12. (holotype), 77B-7-4, 100-102 cm, Cse.1, H24/4 (150X).

13. 77B-7-4, 100-102 cm, Cse.1, A46/1 (150X).

14. 73-7-1, 80-82 cm, Cse.1, U26/2 (90X). 
PLATE 1
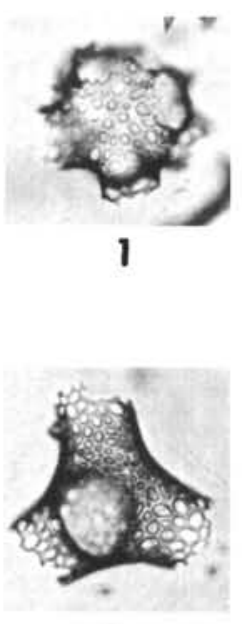

2

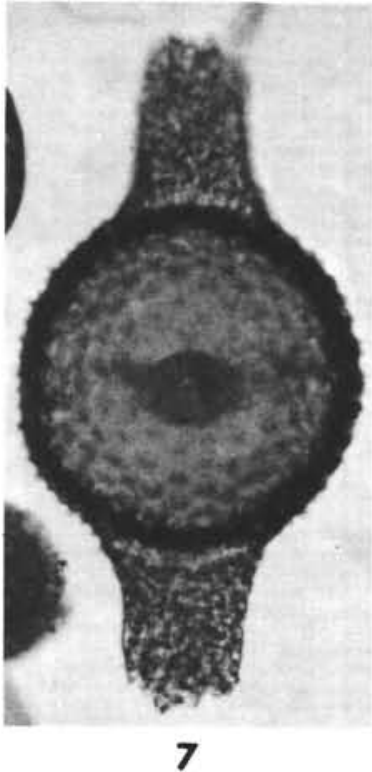

8
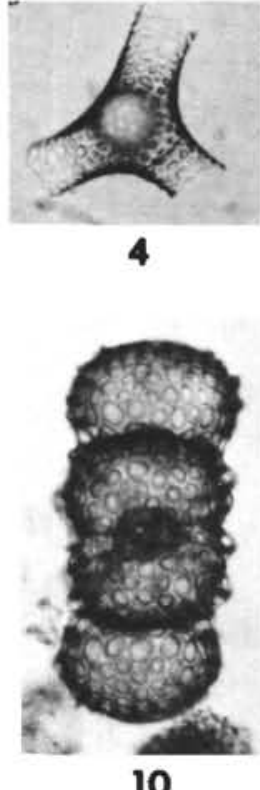

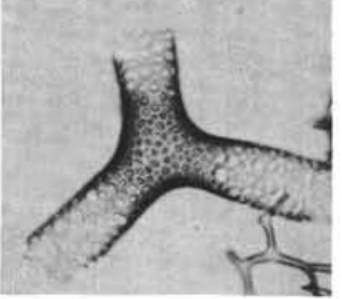

5

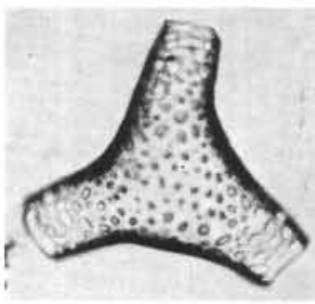

6
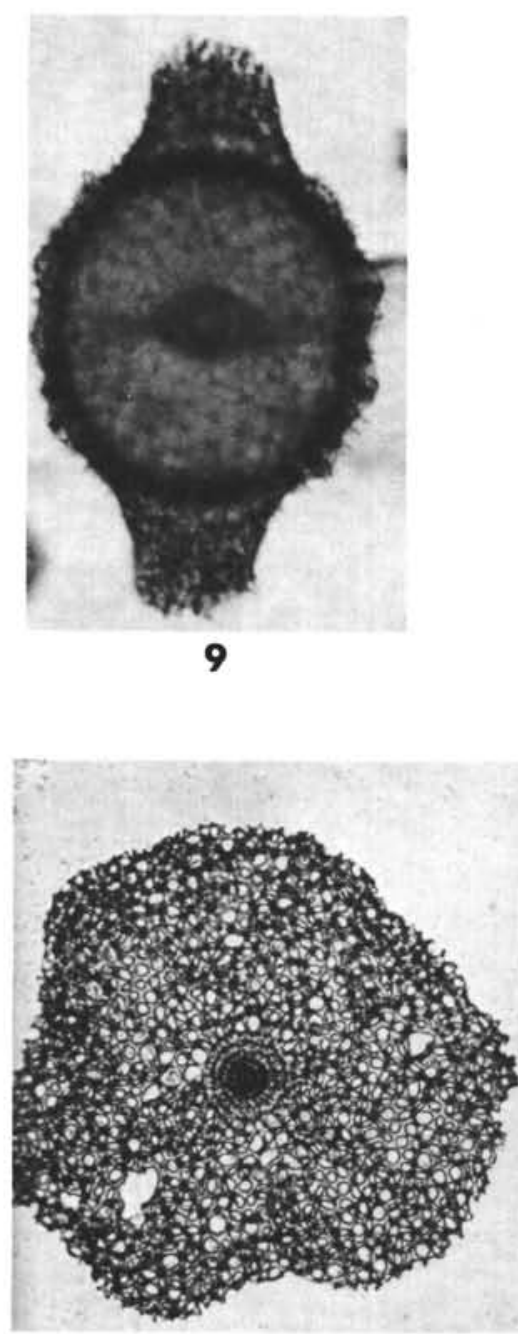

13

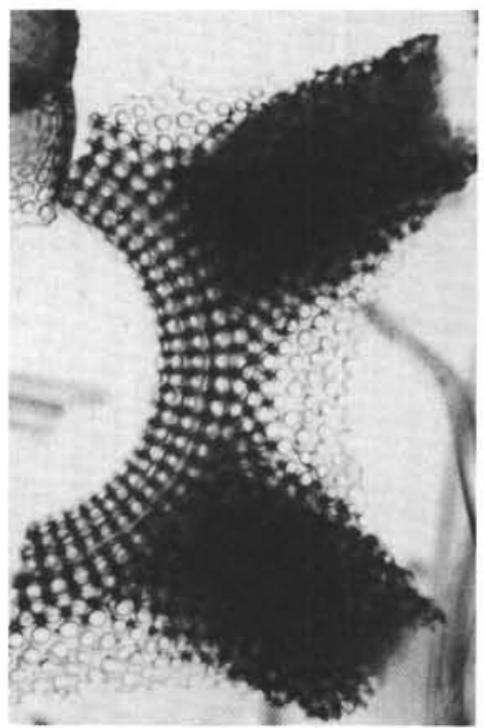

11

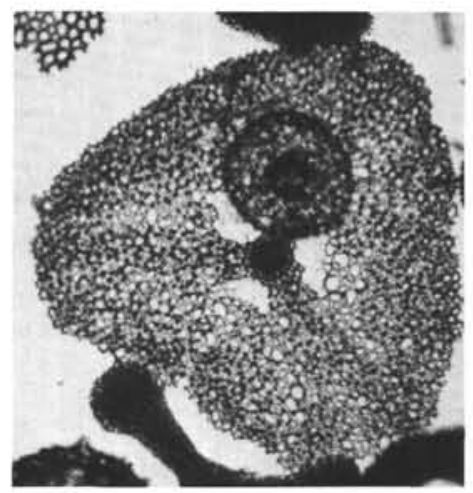

14 
PLATE 2

(Unless otherwise indicated, magnifications are 255X)

Figure $1 \quad$ Nephrospyris renilla 238-6-4, 98-101 cm, Cse.1, P15/2 (180X).

Figure 2 Artophormis sp. cf. A. gracilis $233 \mathrm{~A}-5-4,105-110 \mathrm{~cm}, \mathrm{Sl} .2, \mathrm{E} 14 / 0$.

Figures 3-5 Bekomiforma mynx Sanfilippo and Riedel, new genus and new species.

3, 4. (holotype), 238-17-6, 99-102 cm, S1.2, N21/0 $(4,480 \mathrm{X})$.

5. 77B-5-6, 130-132 cm, Cse.1, W14/3.

Figures 6, 7 Theoperids sp., cf. Bekomiforma mynx.

6. 233-10-6, 110-112 cm, Sl.2, M16/4.

7. $233-18-5,110-112 \mathrm{~cm}, \mathrm{Ph} .2, \mathrm{~T} 15 / 2$.

Figure $8 \quad$ Lychnodictyum audax

238-19-1, 48-51 cm, Cse.1, M14/2. 
PLATE 2

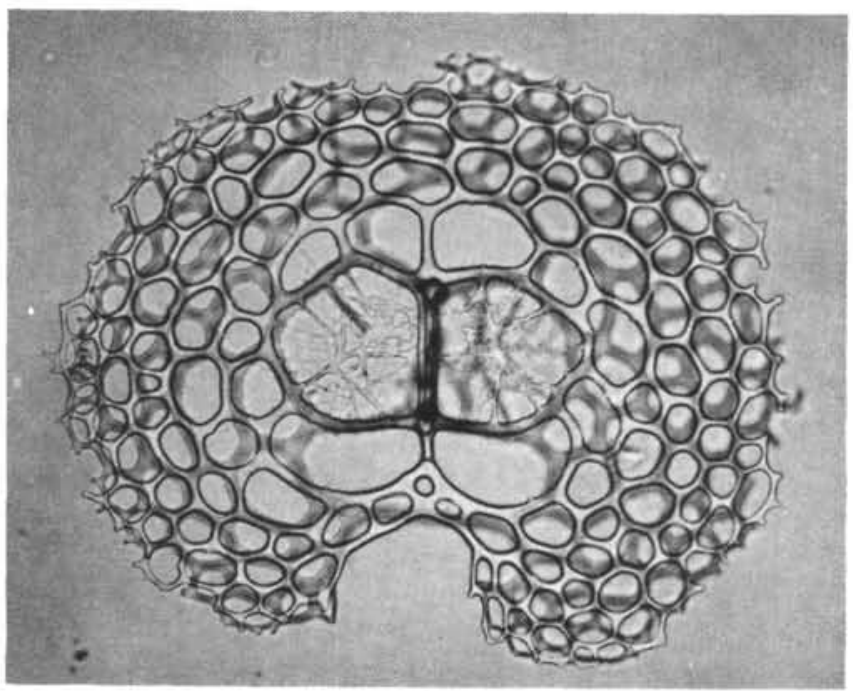

1

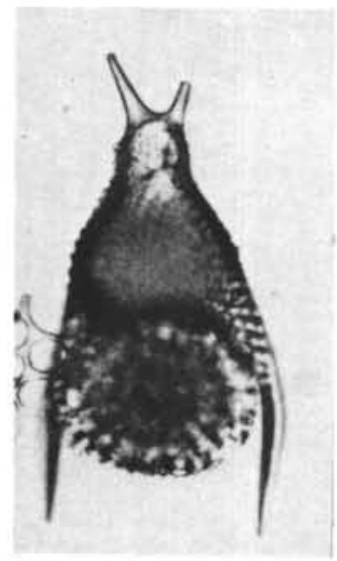

3

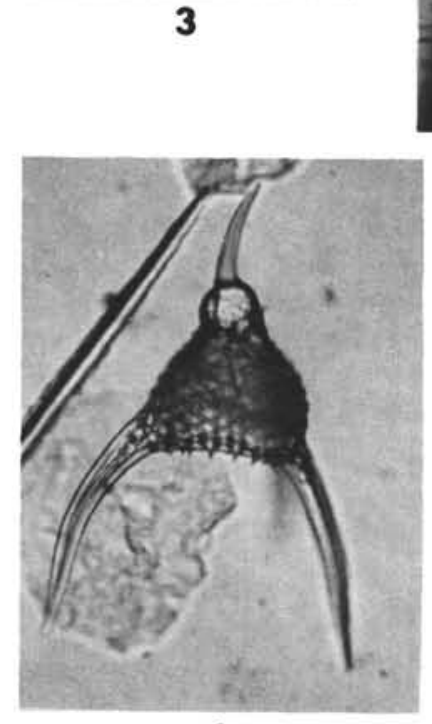

6

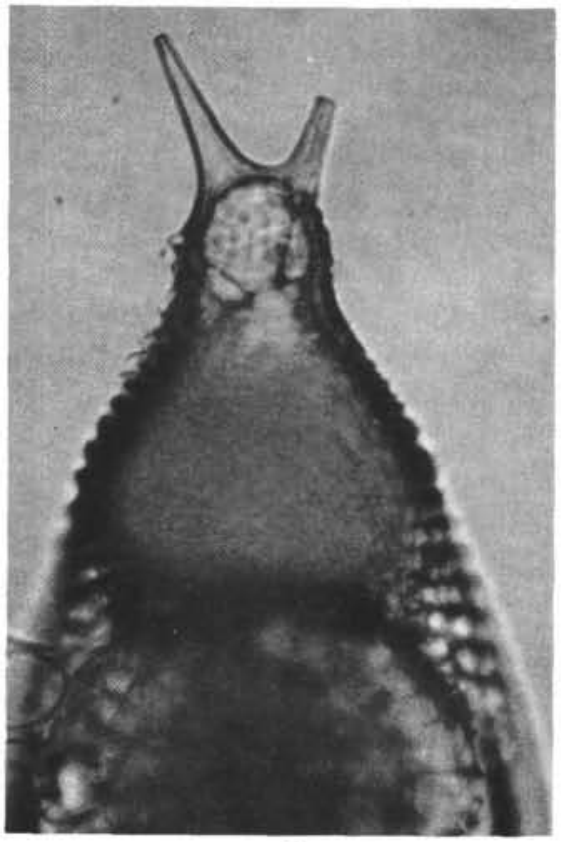

4

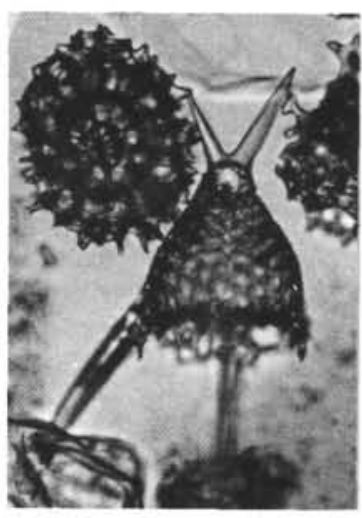

7

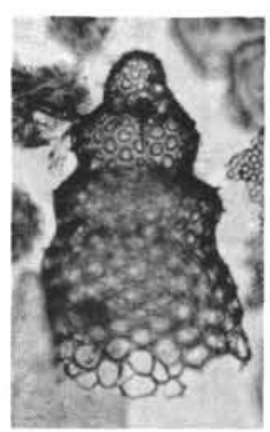

2

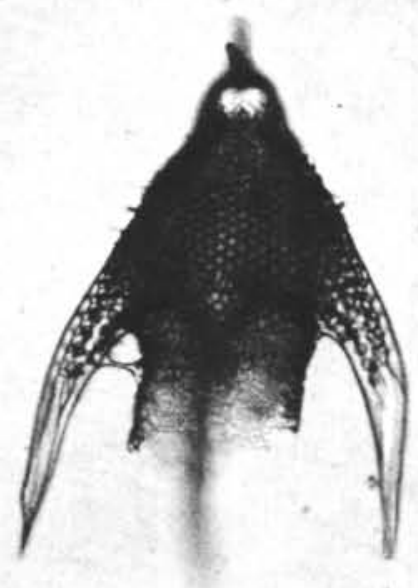

5

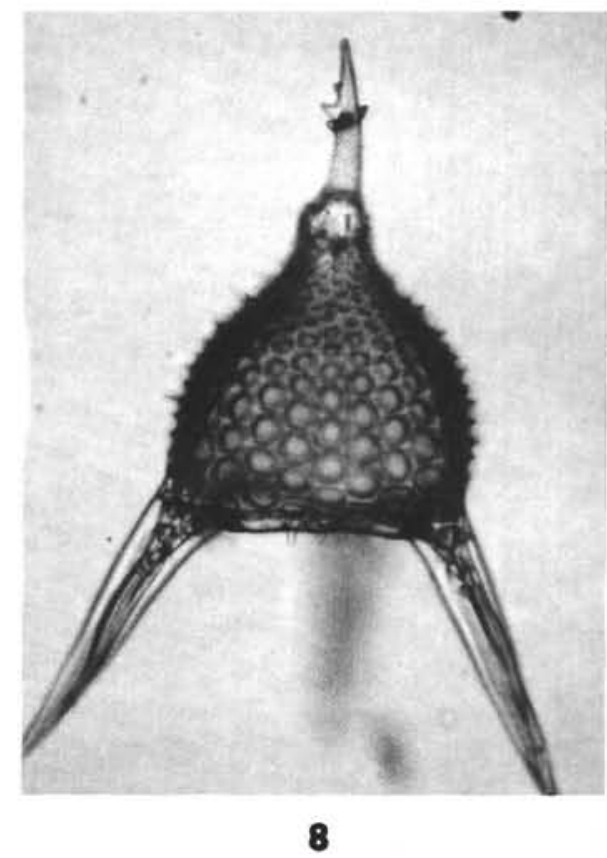




\section{PLATE 3}

(Unless otherwise indicated, magnifications are 255X).

Figures 1-3 Theoperid sp. cf. Theocorys anapographa.

1. 237-30-1, 50-52 cm, Cse.1, P30/3.

2. 237-30-1, 50-52 cm, Cse.1, K41/1.

3. 237-30-1, 50-52 cm, Cse.2, K15/0.

Figure $4 \quad$ Tricolocampe vitrea 237-37-1, approx. $133 \mathrm{~cm}, \mathrm{Sl} .1, \mathrm{~T} 37 / 1$.

Figures 5, 6 Theoperid gen. et sp. indet. Provocative cephalis with three small horns.
5. 237-25-1, 48-52 cm, Cse.1, R18/2.
6. 237-25-1, 48-52 cm, Sl.1, Q5/0 (480X).

Figure 7 Carpocanopsis cingulata

238-27-6, 95-103 cm, S1.1, S26/3.

Figure $8 \quad$ Calocycletta caepa

73-8-4, 80-82 cm, Cse.1, Q39/2.

Figures 9, 10 Lamprocyrtis haysi.

9. $73-2-3,82-84 \mathrm{~cm}, \mathrm{Ph} .2, \mathrm{~B} 45 / 3$.

10. $73-2-3,82-84 \mathrm{~cm}, \mathrm{Ph} .1, \mathrm{X} 17 / 3$.

Figure 11 Lamprocyrtis neoheteroporos.

73-4-6, 130-132 cm, Sl.2, Y33/2.

Figures 12-14 Pterocorys hertwigii.

12. 77A-1-1, 75-77 cm, Ph.1, E24/2.

13. 77A-1-1, $75-77 \mathrm{~cm}$, Cse.1, M31/3.

14. 77A-1-1, 75-77 cm, Ph.1, R25/4. 
PLATE 3

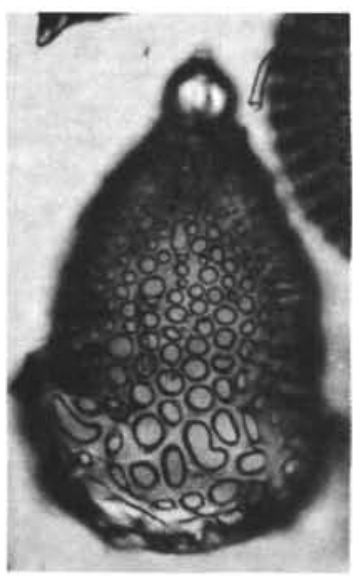

I

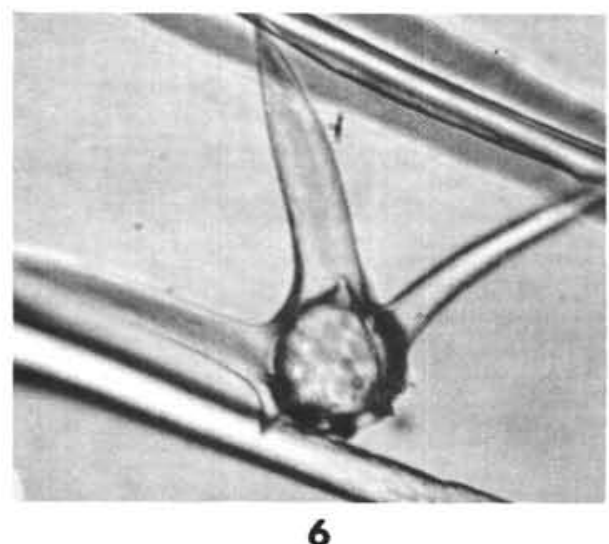

6
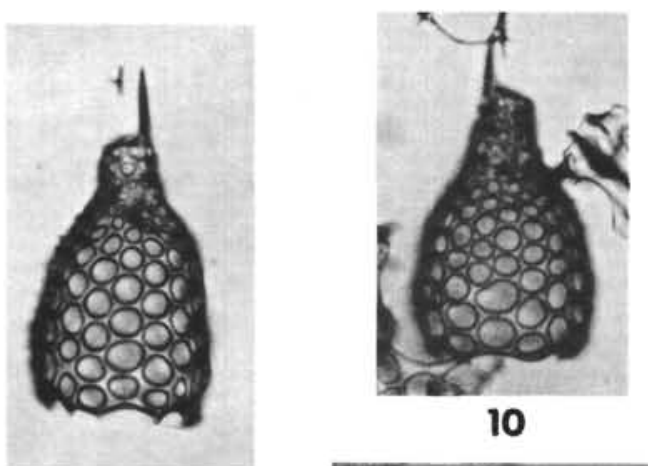

9

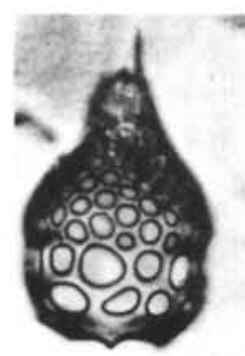

11

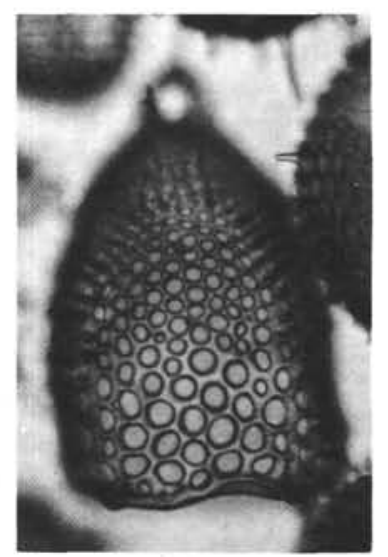

2

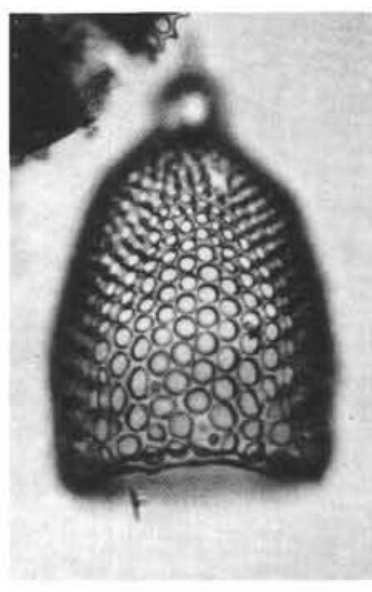

3

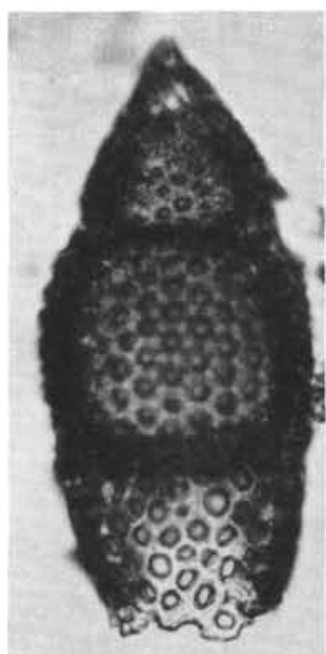

4
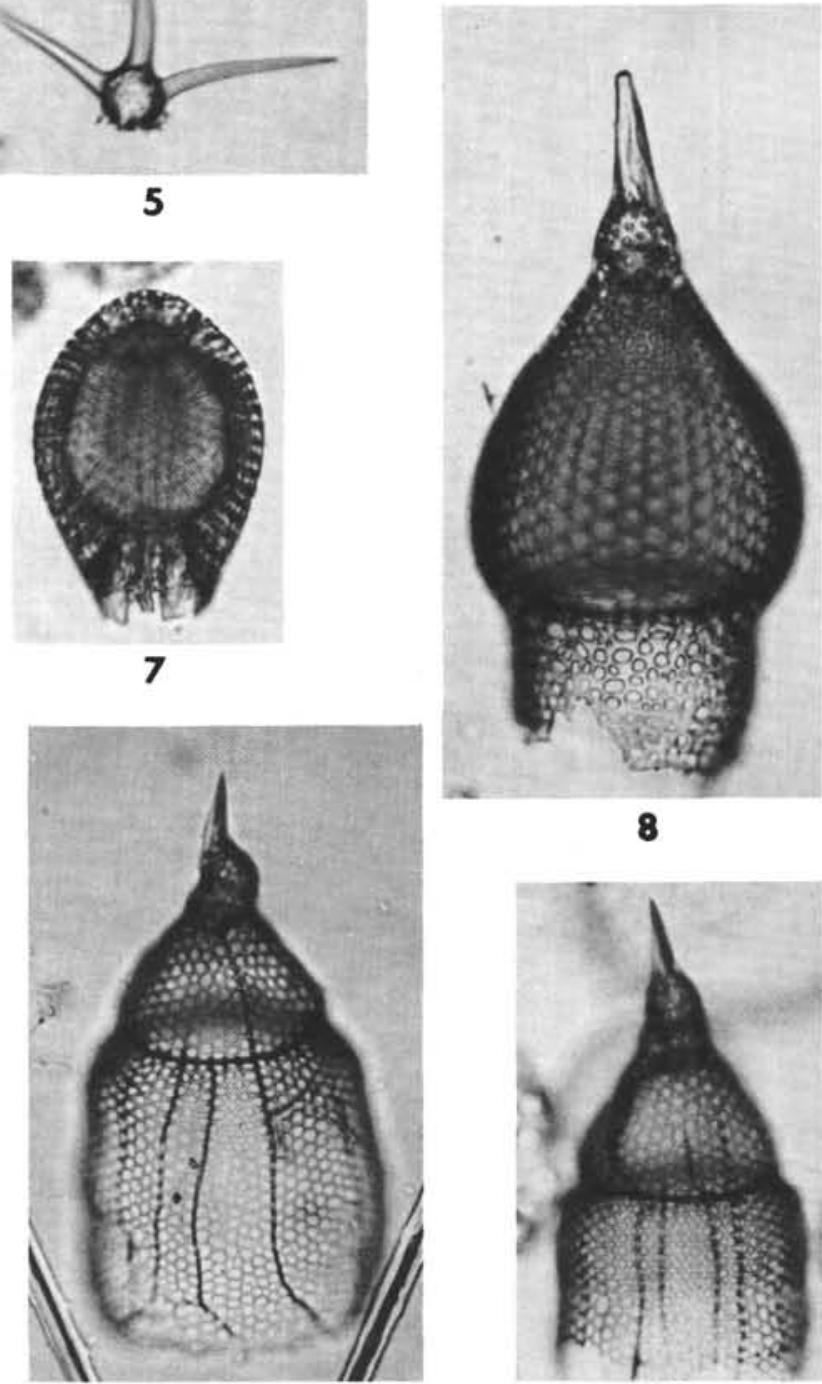

13

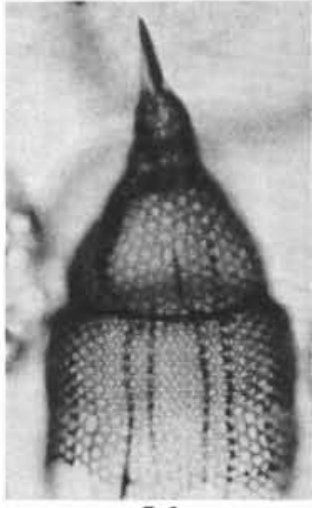

14 


\section{PLATE 4}

(All magnifications are 255X)

Figures 1,2 Pterocorys sp. aff. $P$. hertwigii, with fewer ribs in the shell wall than that species.

1. 77B-3-1, 25-26 cm, Cse. $2,035 / 3$.

2. 77B-2-6, 60-62 cm, Cse.1, J46/4.

Figures 3-5 Theocorythium trachelium.

3. 238-3-6, 125-127 cm, Ph.1, J42/3.

4. 73-3-6, 130-132 cm, Cse.2, D39/2.

5. 238-3-6, 125-127 cm, Ph.1, V19/3.

Figures 6, 7 Theocorythium vetulum.

6. 73-3-6, 130-132 cm, Cse.2, H8/3.

7. 77B-2-6, 60-62 cm, Cse.1, H38/3.

Figures 8,9 Artostrobium rhinoceros Sanfilippo and Riedel, new species.

8. (holotype), 73-5-1, 98-100 cm, Ph.2, L11/0.

9. 233-10-6, 110-112 cm, Ph.1, L36/1.

Figures 10, 11 Artostrobiids cf. Artostrobium rhinoceros.

10. $233-7-5,110-112 \mathrm{~cm}, \mathrm{Ph} .1, \mathrm{~V} 32 / 1$.

11. 233-12-6, 110-114 cm, Ph.1, K26/1. 

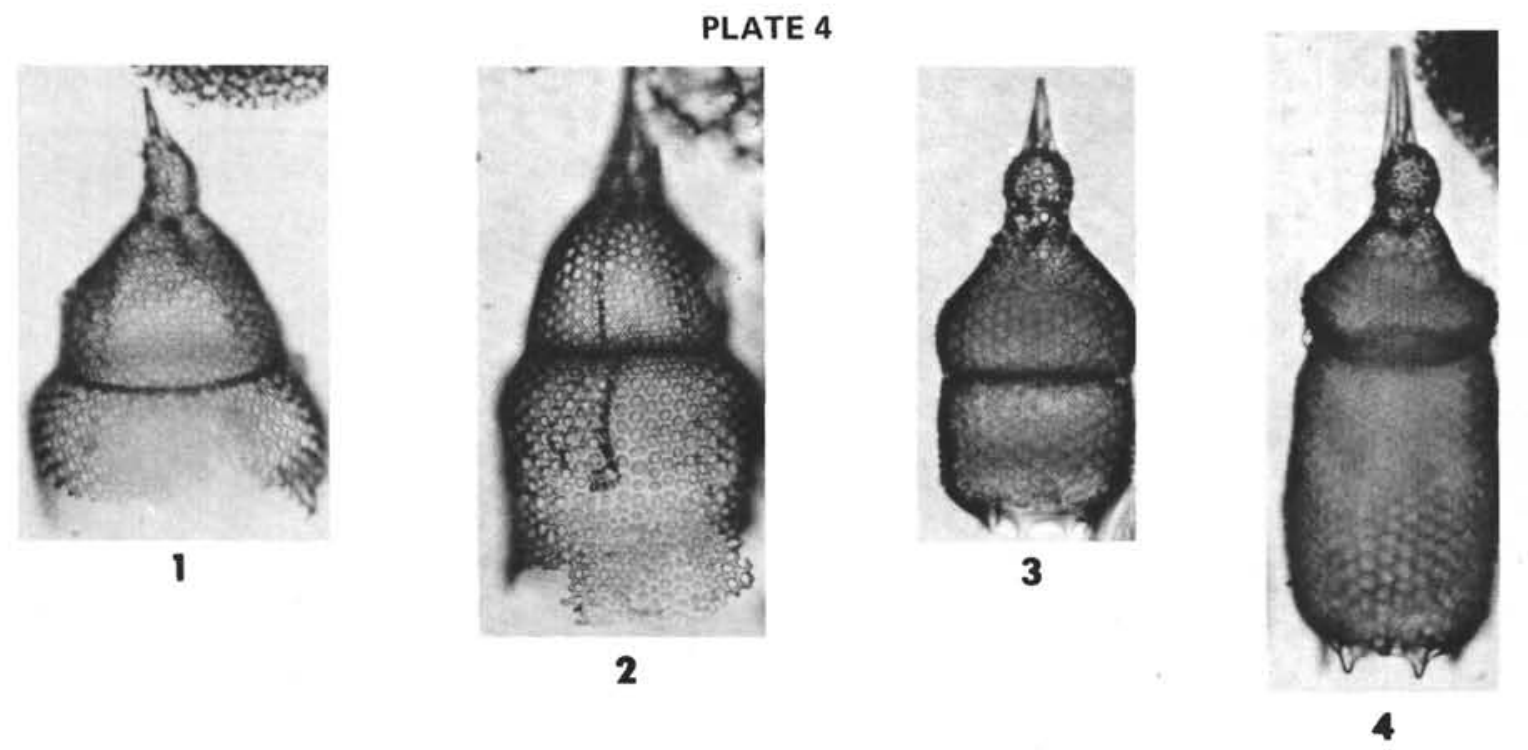

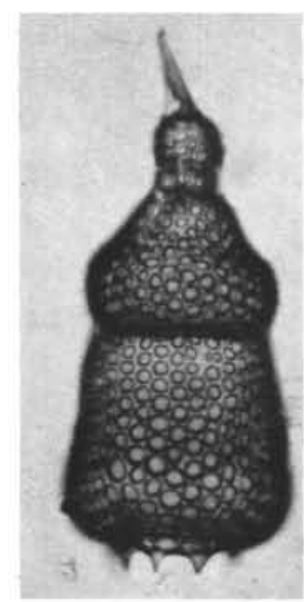

5

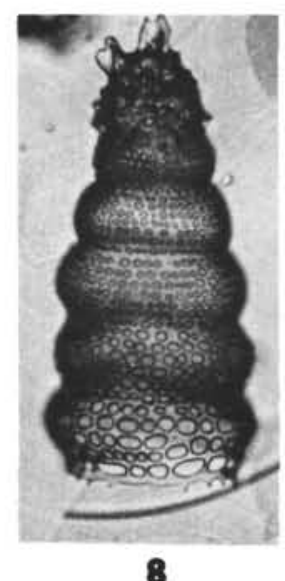

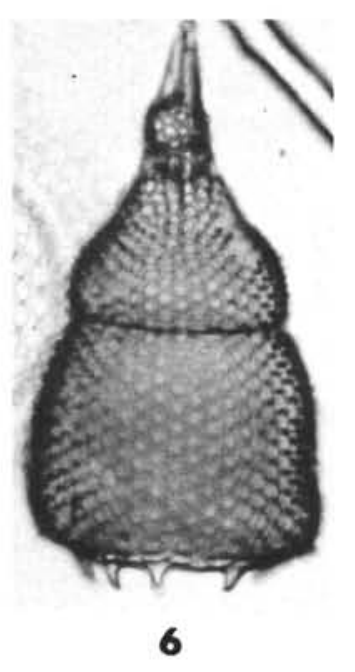
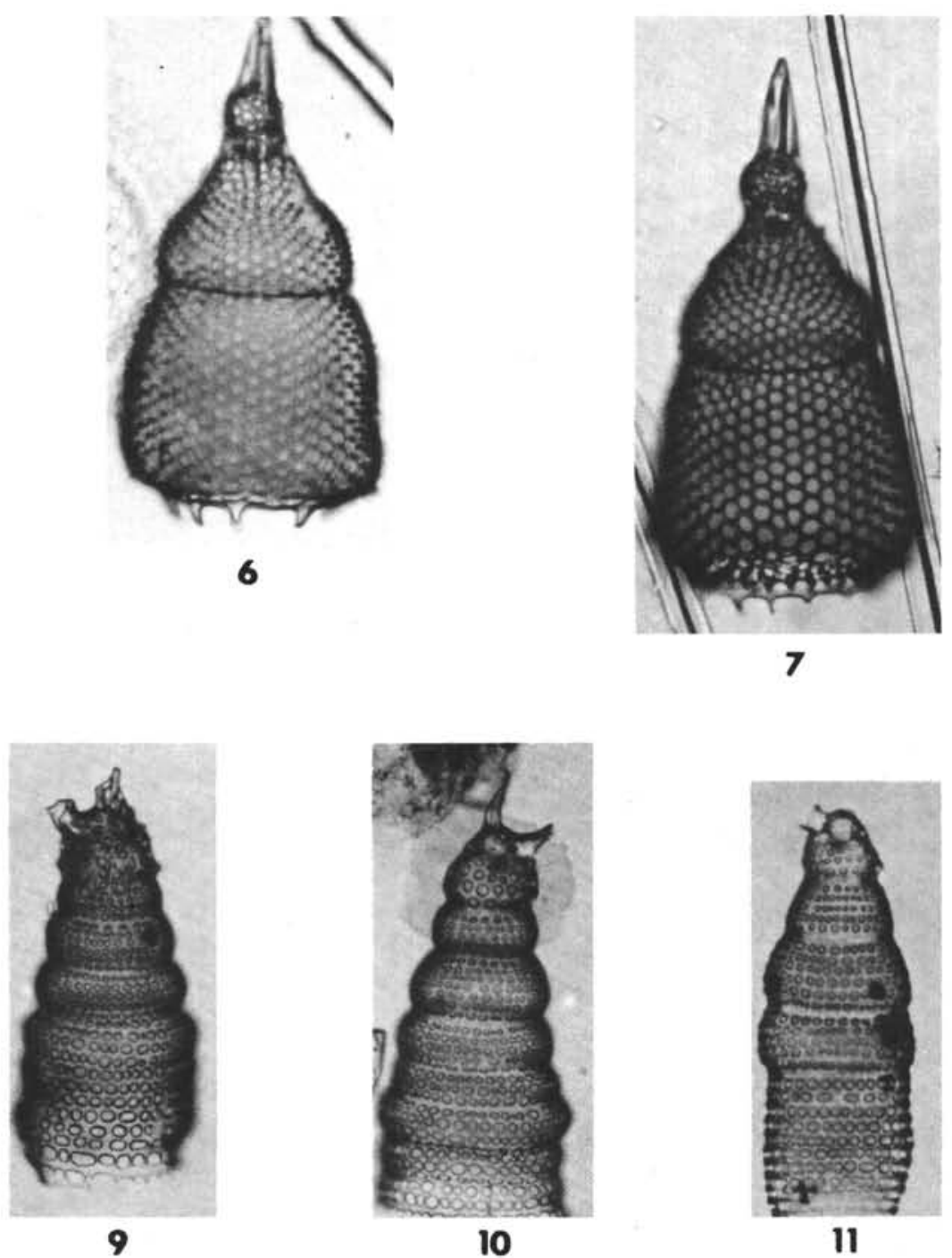